\title{
SARS-CoV-2 mRNA vaccines induce persistent human germinal centre responses
}

https://doi.org/10.1038/s41586-021-03738-2

Received: 8 March 2021

Accepted: 18 June 2021

Published online: 28 June 2021

Check for updates

\author{
Jackson S. Turner, ${ }^{1,12}$, Jane A. O'Halloran ${ }^{2,12}$, Elizaveta Kalaidina ${ }^{3}$, Wooseob Kim', \\ Aaron J. Schmitz', Julian Q. Zhou', Tingting Lei', Mahima Thapa', Rita E. Chen ${ }^{1,4}$, \\ James Brett Case ${ }^{4}$, Fatima Amanat ${ }^{5,6}$, Adriana M. Rauseo ${ }^{2}$, Alem Haile ${ }^{7}$, Xuping Xie ${ }^{8}$, \\ Michael K. Klebert ${ }^{7}$, Teresa Suessen ${ }^{9}$, William D. Middleton ${ }^{9}$, Pei-Yong Shi ${ }^{8}$, \\ Florian Krammer ${ }^{5}$, Sharlene A. Teefey ${ }^{9}$, Michael S. Diamond ${ }^{4,10,11}$, Rachel M. Presti ${ }^{2,10} \bowtie$ \& \\ Ali H. Ellebedy $\mathbf{y}^{1,10,11 凶}$
}

SARS-CoV-2 mRNA-based vaccines are about 95\% effective in preventing COVID-19 ${ }^{1-5}$. The dynamics of antibody-secreting plasmablasts and germinal centre B cells induced by these vaccines in humans remain unclear. Here we examined antigen-specific B cell responses in peripheral blood $(n=41)$ and draining lymph nodes in 14 individuals who had received 2 doses of BNT162b2, an mRNA-based vaccine that encodes the full-length SARS-CoV-2 spike (S) gene ${ }^{1}$. Circulating IgG- and IgA-secreting plasmablasts that target the $S$ protein peaked one week after the second immunization and then declined, becoming undetectable three weeks later. These plasmablast responses preceded maximal levels of serum anti-S binding and neutralizing antibodies to an early circulating SARS-CoV-2 strain as well as emerging variants, especially in individuals who had previously been infected with SARS-CoV-2 (who produced the most robust serological responses). By examining fine needle aspirates of draining axillary lymph nodes, we identified germinal centre B cells that bound $S$ protein in all participants who were sampled after primary immunization. High frequencies of S-binding germinal centre B cells and plasmablasts were sustained in these draining lymph nodes for at least 12 weeks after the booster immunization. S-binding monoclonal antibodies derived from germinal centre B cells predominantly targeted the receptor-binding domain of the S protein, and fewer clones bound to the $\mathrm{N}$-terminal domain or to epitopes shared with the $\mathrm{S}$ proteins of the human betacoronaviruses OC43 and HKU1. These latter cross-reactive B cell clones had higher levels of somatic hypermutation as compared to those that recognized only the SARS-CoV-2 S protein, which suggests a memory B cell origin. Our studies demonstrate that SARS-CoV-2 mRNA-based vaccination of humans induces a persistent germinal centre $B$ cell response, which enables the generation of robust humoral immunity.
The concept of using mRNAs as vaccines was introduced over 30 years ago $^{6,7}$. Key refinements that improved the biological stability and translation capacity of exogenous mRNA enabled development of these molecules as vaccines ${ }^{8,9}$. The emergence of SARS-CoV- 2 in December 2019 , and the ensuing pandemic, has revealed the potential of this platform $^{9-11}$. Hundreds of millions of people have received one of the two SARS-CoV-2 mRNA-based vaccines that were granted emergency use authorization by the US Food and Drug Administration in December
2020. Both of these vaccines demonstrated notable immunogenicity in phase-I/II studies and efficacy in phase-III studies ${ }^{1-4,12-14}$. Whether these vaccines induce the robust and persistent germinal centre reactions that are critical for generating high-affinity and durable antibody responses has not been examined in humans. To address this question, we conducted an observational study of 41 healthy adults ( 8 of whom had a history of confirmed SARS-CoV-2 infection) who received the Pfizer-BioNTech SARS-CoV-2 mRNA vaccine BNT162b2 (Extended Data

'Department of Pathology and Immunology, Washington University School of Medicine, St Louis, MO, USA. ${ }^{2}$ Division of Infectious Diseases, Department of Internal Medicine, Washington University School of Medicine, St Louis, MO, USA. ${ }^{3}$ Division of Allergy and Immunology, Department of Internal Medicine, Washington University School of Medicine, St Louis, MO, USA.

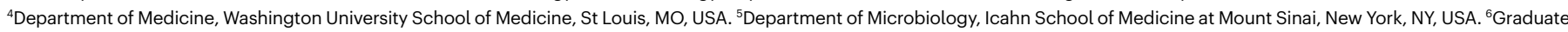
School of Biomedical Sciences, Icahn School of Medicine at Mount Sinai, New York, NY, USA. ${ }^{7}$ Clinical Trials Unit, Washington University School of Medicine, St Louis, MO, USA. ${ }^{8}$ University of

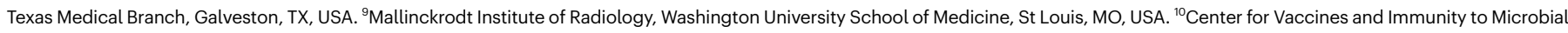
Pathogens, Washington University School of Medicine, St Louis, MO, USA. "The Andrew M. and Jane M. Bursky Center for Human Immunology \& Immunotherapy Programs, Washington University School of Medicine, St Louis, MO, USA. ${ }^{12}$ These authors contributed equally: Jackson S. Turner, Jane A. O’Halloran. ${ }^{\bowtie}$ e-mail: prestir@wustl.edu; ellebedy@wustl.edu 
a

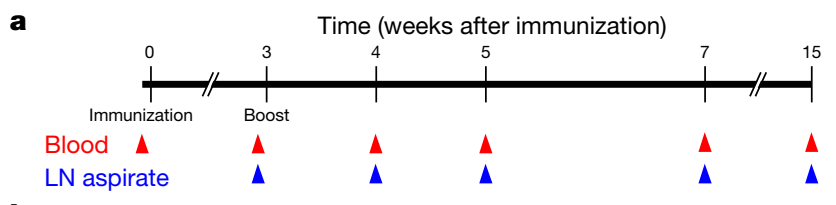

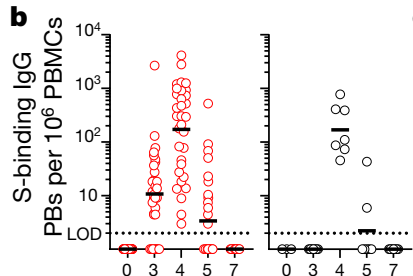

Time (weeks after immunization)

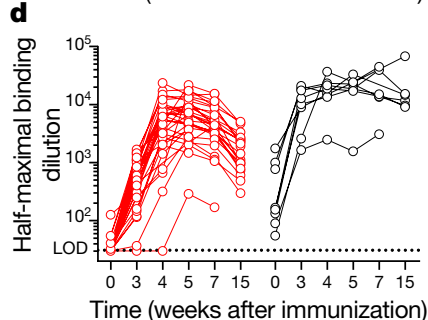

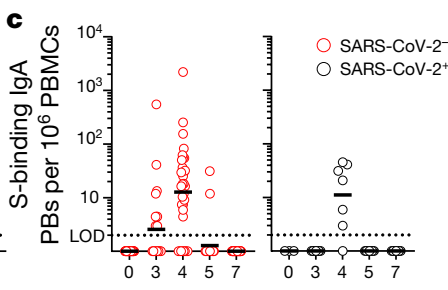

Time (weeks after immunization)

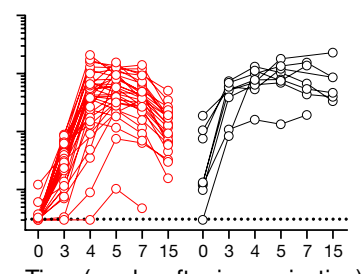

Time (weeks after immunization)
Fig. 1 | Plasmablast and antibody response to SARS-CoV-2 immunization. a, Study design. Forty-one healthy adult volunteers (ages 28-73, 8 with a history of SARS-CoV-2 infection) were enrolled and received the BNT162 b2 mRNA SARS-CoV-2 vaccine. Blood was collected before immunization, and at 3, 4, 5, 7 and 15 weeks after immunization. For 14 participants (ages 28-52, none with a history of SARS-CoV-2 infection), FNAs of ipsilateral axillary lymph nodes (LNs) were collected at 3, 4, 5, 7 and 15 weeks after immunization.b, c, ELISpot quantification of S-binding IgG-(b) and IgA- (c) secreting plasmablasts (PBs) in blood at baseline, and at $3,4,5$ and 7 weeks after immunization in participants without (red) and with (black) a history of SARS-CoV-2 infection. d, Plasma IgG titres against SARS-CoV-2 S (left) and the RBD of S (right) measured by ELISA in participants without (red) and with (black) a history of SARS-CoV-2 infection at baseline, and at 3, 4, 5, 7 and 15 weeks after immunization. Dotted lines indicate limits of detection. Symbols at each time point in $\mathbf{b}-\mathbf{d}$ represent one sample $(n=41)$. Results are from one experiment performed in duplicate.

Tables 1,2). Blood samples were collected at baseline, and at weeks 3 (pre-boost), 4, 5, 7 and 15 after the first immunization. Fine needle aspirates (FNAs) of the draining axillary lymph nodes were collected from 14 participants (none with history of SARS-CoV-2 infection) at weeks 3 (pre-boost), 4, 5, 7, and 15 after the first immunization (Fig. 1a).

We used an enzyme-linked immune absorbent spot (ELISpot) assay to measure antibody-secreting plasmablasts in blood that bound SARS-CoV-2 S protein. We detected SARS-CoV-2-S-specfic IgG- and IgA-secreting plasmablasts 3 weeks after primary immunization in 24 of 33 participants with no history of SARS-CoV-2 infection, but in 0 of 8 participants who had previously been infected with SARS-CoV-2. Plasmablasts peaked in blood during the first week after boosting (week 4 after primary immunization), with frequencies that varied widely from 3 to $4,100 \mathrm{~S}$-binding plasmablasts per $10^{6}$ peripheral blood mononuclear cells (PBMCs) (Fig. 1b, c). We found that plasma IgG antibody titres against $\mathrm{S}$, measured by enzyme-linked immunosorbent assay (ELISA), increased in all participants over time, and reached peak geometric mean half-maximal binding titres of 5,567 and 15,850 at 5 weeks after immunization among participants without and with history of SARS-CoV-2 infection, respectively, with a subsequent decline by 15 weeks after immunization. Anti-S IgA titres and IgG titres against the receptor-binding domain (RBD) of S showed similar kinetics, and reached peak geometric mean half-maximal binding titres of 172 and 739 for anti-S IgA and 4,501 and 7,965 for anti-RBD IgG among participants without and with history of SARS-CoV-2 infection, respectively, before declining. IgM responses were weaker and more transient, peaking 4 weeks after immunization among participants without history of SARS-CoV-2 infection with a geometric mean half-maximal binding titre of 78 and were undetectable in all but 2 previously infected participants (Fig. 1d, Extended Data Fig. 1a).

The functional quality of serum antibody was measured using high-throughput focus reduction neutralization tests ${ }^{15}$ on Vero cells expressing TMPRSS2 against three authentic infectious SARS-CoV-2 strains with sequence variations in the $S$ gene $^{16,17}$ : (1) a Washington strain (2019n-CoV/USA) with a prevailing D614G substitution (WA1/2020 D614G); (2) a B.1.1.7 isolate with signature changes in the $S$ gene ${ }^{18}$, including mutations resulting in the deletion of residues $69,70,144$ and 145 as well as N501Y, A570D, D614G and P681H substitutions; and (3) a chimeric SARS-CoV-2 with a B.1.351S gene in the Washington strain background (Wash-B.1.351) that contained the following changes: D80A, deletion of residues 242-244, R246I, K417N, E484K, N501Y, D614G and A701V. Serum neutralizing titres increased markedly in participants without a history of SARS-CoV-2 infection after boosting, with geometric mean neutralization titres against WA1/2020 D614G of 58 at 3 weeks after primary immunization and 572 at 2 or 4 weeks after boost ( 5 or 7 weeks after primary immunization). Neutralizing titres against the B.1.1.7 and B.1.351 variants were lower, with geometric mean neutralization titres of 49 and 373 against B.1.1.7 and 36 and 137 against B.1.351 after primary and secondary immunization, respectively. In participants with a history of previous SARS-CoV-2 infection, neutralizing titres against all three viruses were detected at baseline (geometric mean neutralization titres of 241.8, 201.8 and 136.7 against WA1/2020 D614G, B.1.1.7 and B.1.351, respectively). In these participants, neutralizing titres increased more rapidly and to higher levels after immunization, with geometric mean neutralization titres of 4,544,3,584 and 1,897 against WA1/2020 D614G, B.1.1.7 and B.1.351, respectively, after primary immunization, and 9,381, 9,351 and 2,749 against WA1/2020 D614G, B.1.1.7 and B.1.351, respectively, after secondary immunization. These geometric mean neutralization titres were 78-, 73- and 53-fold higher after primary immunization and 16-, 25- and 20 -fold higher after boosting against WA1/2020 D614G, B.1.1.7 and B.1.351, respectively, than in participants without a history of SARS-CoV-2 infection (Extended Data Fig. 1b).

The BNT162b2 vaccine is injected into the deltoid muscle, which drains primarily to the lateral axillary lymph nodes. We used ultrasonography to identify and guide FNA of accessible axillary nodes on the side of immunization approximately 3 weeks after primary immunization. In 5 of the 14 participants, a second draining lymph node was identified and sampled after secondary immunization (Fig. 2a). Germinal centre B cells (defined as $\mathrm{CD} 19^{+} \mathrm{CD} 3^{-} \mathrm{IgD}^{\text {low }} \mathrm{BCL} 6^{+} \mathrm{CD} 38^{\text {int }} \mathrm{lym}$ phocytes) were detected in all lymph nodes (Fig. 2b, d, Extended Data Fig. 2a, Extended Data Table 3). We co-stained FNA samples with two fluorescently labelled S probes to detect S-binding germinal centre B cells. A control tonsillectomy sample with a high frequency of germinal centre B cells that was collected before the COVID-19 pandemic from an unrelated donor was stained as a negative control. S-binding germinal centre B cells were detected in FNAs from all 14 participants following primary immunization. The kinetics of the germinal centre response varied among participants, but S-binding germinal centre B cell frequencies increased at least transiently in all participants after boosting and persisted at high frequency in most individuals for at least 7 weeks. Notably, S-binding germinal centre B cells remained at or near their peak frequency 15 weeks after immunization in 8 of the 10 participants sampled at that time point, and these prolonged germinal centre responses had high proportions of S-binding cells (Fig. 2c-e, Extended Data Fig. 2b).

To evaluate the domains targeted by the S-protein-specific germinal centre response after vaccination, we generated recombinant monoclonal antibodies from single-cell-sorted S-binding germinal centre $\mathrm{B}$ cells (defined by the surface-marker phenotype $\mathrm{CD} 19^{+} \mathrm{CD}^{-}$ IgD ${ }^{\text {low }} \mathrm{CD} 20^{\text {high }} \mathrm{CD} 38^{\text {int }} \mathrm{CD} 71^{+} \mathrm{CXCR} 5^{+}$lymphocytes) from three of the participants one week after boosting (Extended Data Fig. 2a). Fifteen, 

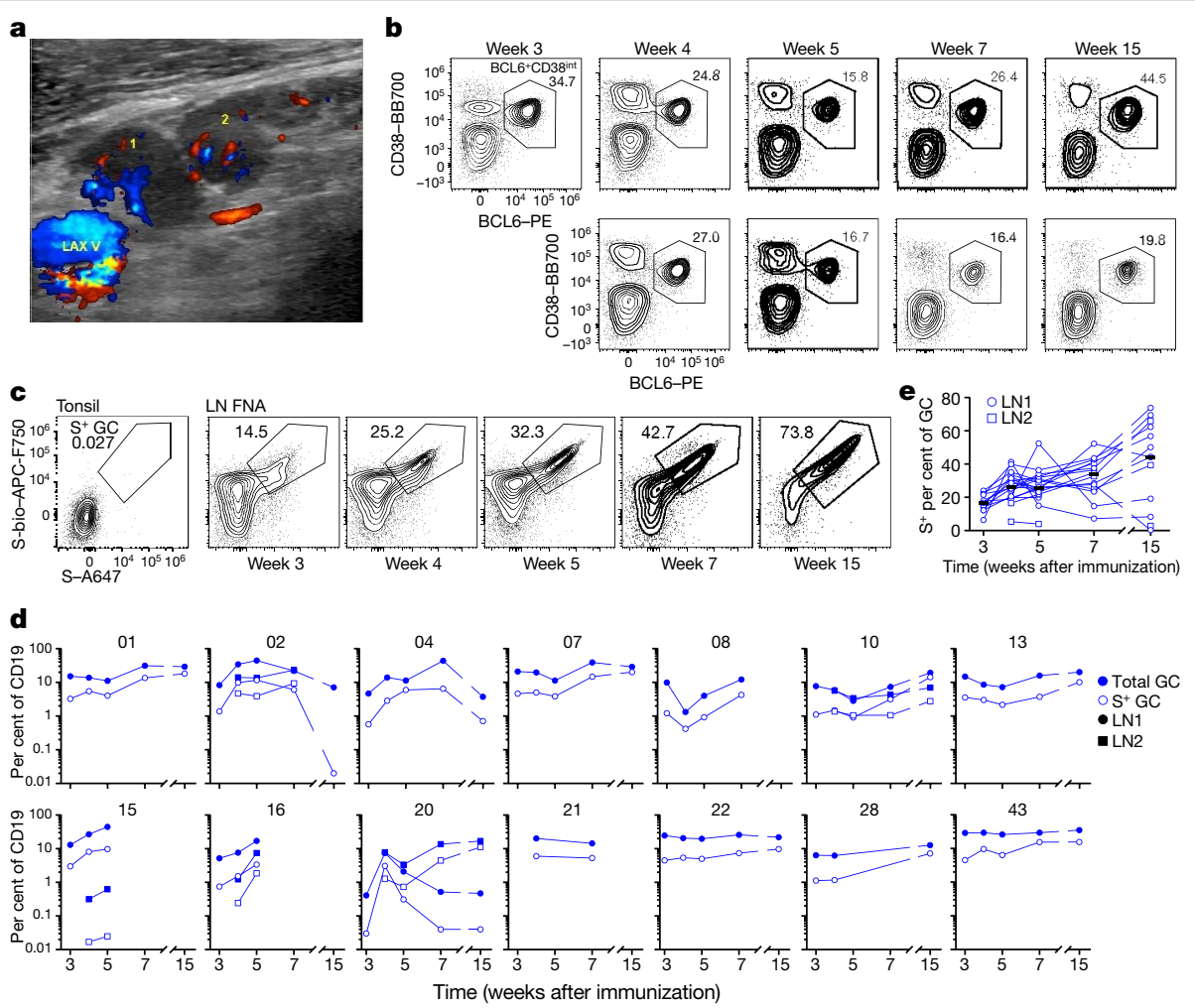

Fig. 2 | Germinal centre B cell response to SARS-CoV-2 immunization. a, Representative colour Doppler ultrasound image of two draining lymph nodes (' 1 ' and ' 2 ') adjacent to the axillary vein 'LAX V' 5 weeks after immunization. b, c, Representative flow cytometry plots of BCL6 and CD38 staining on IgD ${ }^{\text {low }} \mathrm{CD} 19^{+} \mathrm{CD}^{-}$live singlet lymphocytes in FNA samples (b; LN1, top row; $\mathrm{LN} 2$, bottom row) and S staining on $\mathrm{BCL} 6^{+} \mathrm{CD} 38^{\text {int }}$ germinal centre $\mathrm{B}$ cells in tonsil and FNA samples (c) at the indicated times after immunization. d, e, Kinetics of total (blue) and $\mathrm{S}^{+}$(white) germinal centre (GC) B cells as gated in $\mathbf{b}$ and $\mathbf{c}(\mathbf{d})$ and S-binding per cent of germinal centre B cells (e) from FNA of draining lymph nodes. Symbols at each time point represent one FNA sample; square symbols denote the second lymph node sampled $(n=14)$. Horizontal lines indicate the median. five and seventeen S-binding, clonally distinct monoclonal antibodies were generated from participants 07,20 (lymph node 1) and 22, respectively (Extended Data Table 4). Of the $37 \mathrm{~S}$-binding monoclonal antibodies, 17 bound the RBD, 6 recognized the $\mathrm{N}$-terminal domain and 3 were cross-reactive with $\mathrm{S}$ proteins from seasonal betacoronavirus OC43; 2 of these monoclonal antibodies also bound $S$ from seasonal betacoronavirus HKU1 (Fig. 3a). Clonal relatives of 14 out of 15,1 out of 5 and 12 out of 17 of the S-binding monoclonal antibodies were identified among bulk-sorted total plasmablasts from PBMCs and germinal centre $B$ cells at 4 weeks after immunization from participants 07,20 and 22, respectively (Fig. 3b, Extended Data Figs. 2c, 3a, b, Extended Data Tables 5, 6). Clones related to S-binding monoclonal antibodies had significantly increased mutation frequencies in their immunoglobulin heavy chain variable region (IGHV) genes compared to previously published naive $B$ cells ${ }^{19}$, particularly those related to monoclonal antibodies that cross-reacted with seasonal betacoronaviruses (Fig. 3c, d).

In addition to germinal centre $B$ cells, we detected robust plasmablast responses in the draining lymph nodes of all 14 participants in the FNA cohort. S-binding plasmablasts (defined as $\mathrm{CD} 19^{+} \mathrm{CD} 3^{-}$ IgD ${ }^{\text {low }} \mathrm{CD} 20^{\text {low }} \mathrm{CD} 38^{+} \mathrm{CD} 71^{+} \mathrm{BLIMP1}^{+}$lymphocytes) were detected in all of the lymph nodes that we sampled, and increased in frequency after boosting (Extended Data Fig. 4a, b). The detected plasmablasts were unlikely to be a contaminant of blood, because CD14 ${ }^{+}$monocyte and/ or granulocyte frequencies were below $1 \%$ in all FNA samples (well below the $10 \%$ threshold that was previously established ${ }^{19}$ ) (Extended Data Table 3). Moreover, S-binding plasmablasts were detected in FNA samples at 5, 7 and 15 weeks after immunization, when they had become undetectable in blood from all participants in the cohort. The vast majority of S-binding lymph node plasmablasts were isotype-switched at $\mathbf{4}$ weeks after primary immunization, and IgA-switched cells accounted for $25 \%$ or more of the plasmablasts in 6 out of 14 participants (Extended Data Fig. 4c, d).

This study evaluated whether SARS-CoV-2 mRNA-based vaccines induce antigen-specific plasmablast and germinal centre $B$ cell responses in humans. The vaccine induced a strong IgG-dominated plasmablast response in blood that peaked one week after the booster immunization. In the draining lymph nodes, we detected robust SARS-CoV-2S-binding germinal centre B cell and plasmablast responses in aspirates from all 14 of the participants. These responses were detectable after the first immunization but greatly expanded after the booster injection. Notably, S-binding germinal centre B cells and plasmablasts persisted for at least 15 weeks after the first immunization (12 weeks after secondary immunization) in 8 of the 10 participants who were sampled at that time point. These responses to mRNA vaccination are superior to those seen after seasonal influenza virus vaccination in humans ${ }^{19}$, in whom haemagglutinin-binding germinal centre B cells were detected in only three out of eight participants. More robust germinal centre responses are consistent with antigen dissemination to multiple lymph nodes and the self-adjuvating characteristics of the mRNA-lipid nanoparticle vaccine platform compared to nonadjuvanted inactivated vaccines used for seasonal influenza virus vaccination $^{7,20,21}$. Our data in humans corroborate reports that demonstrate the induction of potent germinal centre responses by SARS-CoV-2 mRNA-based vaccines in mice 22,23. $^{2}$.

To our knowledge, this is the first study to provide direct evidence for the induction of a persistent antigen-specific germinal centre B cell response after vaccination in humans. Dynamics of germinal centre B cell responses vary widely depending on the model system in which they are studied, although the most active period of the response usually occurs over the course of a few weeks. Primary alum-adjuvanted protein 

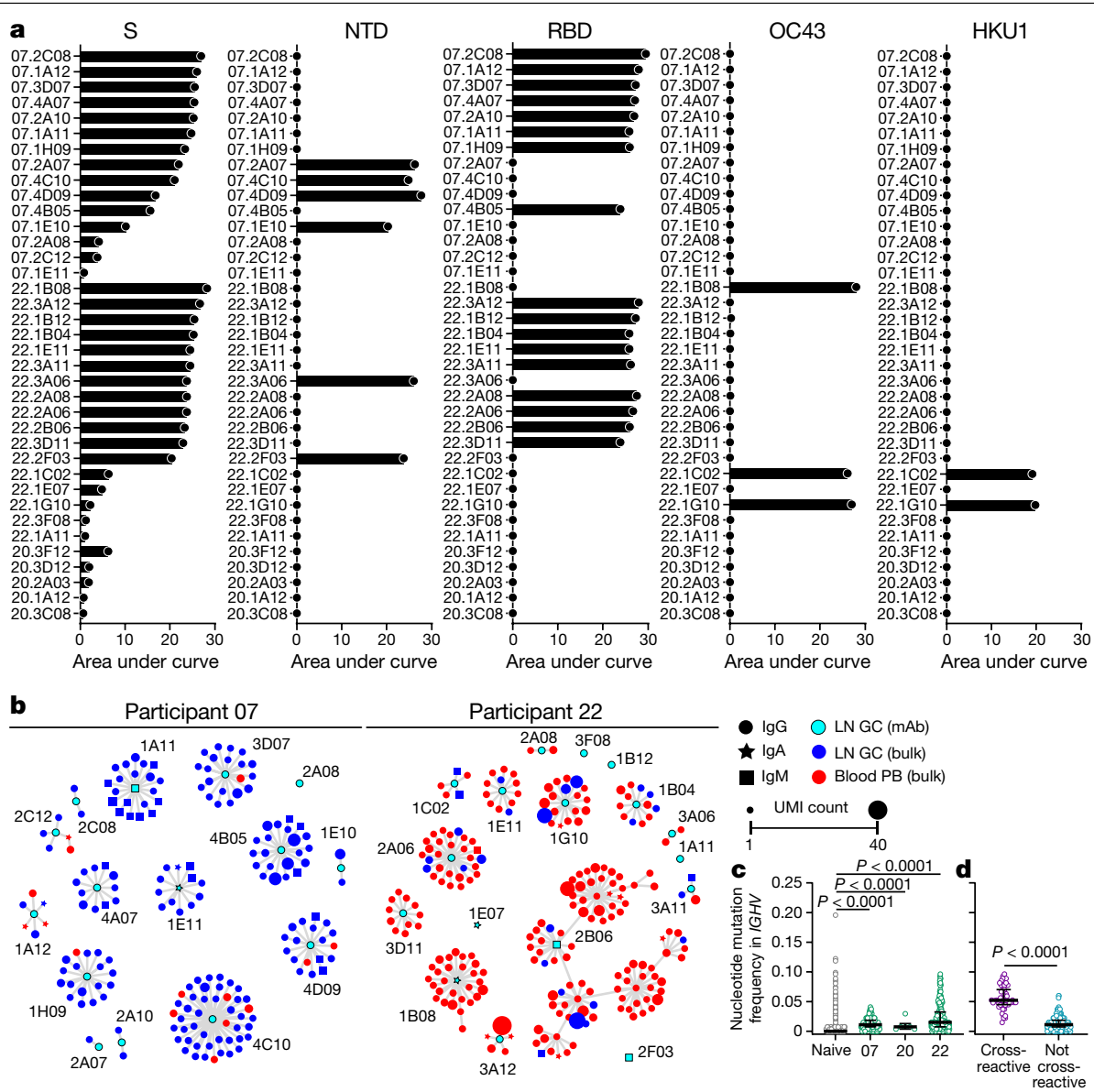

Fig. 3 Clonal analysis of germinal centre response to SARS-CoV-2

immunization. a, Binding of monoclonal antibodies ( $\mathrm{mAbs}$ ) generated from germinal centre $B$ cells to SARS-CoV-2S, $\mathrm{N}$-terminal domain (NTD) of S, RBD, or S proteins of betacoronavirus OC 43 or HKU1, measured by ELISA. Results are from one experiment performed in duplicate. Baseline for area under the curve was set to the mean + three times the s.d. of background binding to bovine serum albumin. $\mathbf{b}$, Clonal relationship of sequences from S-binding germinal centre-derived monoclonal antibodies (cyan) to sequences from bulk repertoire analysis of plasmablasts from PBMCs (red) and germinal centre B cells (blue) sorted 4 weeks after immunization. Each clone is visualized as a network in which each node represents a sequence and sequences are linked as a minimum spanning tree of the network. Symbol shape indicates sequence isotype: IgG (circle), IgA (star) and IgM (square); symbol size corresponds to sequence count. c, d, Comparison of nucleotide mutation frequency in $/ G H V$ genes of naive B cells sorted from individuals vaccinated with influenza virus vaccine $^{19}$ (grey) to clonal relatives of S-binding monoclonal antibodies among plasmablasts sorted from PBMCs and germinal centre B cells 4 weeks after immunization (green) in indicated participants (c) and between clonal relatives of S-binding monoclonal antibodies cross-reactive (purple) or not (teal) to seasonal coronavirus $S$ proteins among plasmablasts sorted from PBMCs and germinal centre B cells 4 weeks after immunization (d). Horizontal lines and error bars indicate the median and interquartile range. Sequence counts were 2,553 (naive), 199 (participant 07), 6 (participant 20), 240 (participant 22), 54 (cross-reactive) and 391 (not cross-reactive). $P$ values from two-sided KruskalWallis test with Dunn's post-test between naive B cells and S-binding clones (c) or two-sided Mann-Whitney $U$ test (d). immunization of mice typically leads to germinal centre responses that peak $1-2$ weeks after immunization and contract at least 10 -fold within 5-7 weeks ${ }^{24-26}$. Germinal centre responses induced by immunization with more robust adjuvants such as sheep red blood cells, complete Freund's adjuvant or saponin-based adjuvants tend to peak slightly later, at 2-4 weeks after vaccination, and can persist at low frequencies for several months ${ }^{27-33}$. Although studies of extended durability are rare, antigen-specific germinal centre B cells have been found to persist for at least one year, albeit at very low levels ${ }^{28,30}$. In this study, we show SARS-CoV-2 mRNA vaccine-induced germinal centre B cells are maintained at or near peak frequencies for at least 12 weeks after secondary immunization.

The persistence of S-binding germinal centre B cells and plasmablasts in draining lymph nodes is a positive indicator for induction of long-lived plasma cell responses ${ }^{25}$. Future studies will be needed to examine whether mRNA vaccination induces a robust $\mathrm{S}$-specific long-lived plasma cell compartment in the bone marrow. As part of such studies, it will be critical to generate a comprehensive set of monoclonal antibodies derived from plasmablasts and germinal centre B cells isolated from several time points to define the breadth of the B cell response elicited by this vaccine. None of the 14 participants in our study who underwent FNA of draining lymph nodes had a history of SARS-CoV-2 infection. Thus, further comparison of vaccine-induced germinal centre responses from naive and previously infected individuals will be informative. Finally, the work presented here focuses on the $\mathrm{B}$ cell component of the germinal centre reaction. A robust $\mathrm{T}$ follicular helper response sustains the germinal centre reaction ${ }^{34,35}$. As such, studies are planned to investigate the magnitude, specificity and durability of the T follicular helper cell response after SARS-CoV-2 mRNA vaccination in humans.

A preliminary observation from our study is the dominance of RBD-targeting clones among responding germinal centre B cells. A more detailed analysis ${ }^{36}$ of these RBD-binding monoclonal antibodies assessed their in vitro inhibitory capacity against the WA1/2020 D614G strain using an authentic SARS-CoV-2 neutralization assay: five showed high neutralization potency, with $80 \%$ neutralization values of less than $100 \mathrm{ng} \mathrm{ml}^{-1}$. For the most part, RBD-binding clones contained few $(<3)$ nonsynonymous nucleotide substitutions in their IGHVgenes, 
which indicates that they originated from recently engaged naive $B$ cells. This contrasts with the three cross-reactive germinal centre B cell clones that recognized conserved epitopes within the S proteins of betacoronaviruses. These cross-reactive clones had significantly higher mutation frequencies, which suggests a memory $B$ cell origin. These data are consistent with previous findings from seasonal influenza virus vaccination in humans that show that the germinal centre reaction can engage pre-existing memory $B$ cells directed against conserved epitopes as well as naive clones targeting novel epitopes ${ }^{19}$. However, these cross-reactive clones were not identified in all individuals and comprised a small fraction of responding B cells, consistent with a similar analysis of SARS-CoV-2 mRNA vaccine-induced plasmablasts ${ }^{37}$. Overall, our data demonstrate the capacity of SARS-CoV-2 mRNA-based vaccines to induce robust and prolonged germinal centre reactions. The induced germinal centre reaction recruited cross-reactive memory $B$ cells as well as newly engaged clones that target unique epitopes within SARS-CoV-2 S protein. Elicitation of high affinity and durable protective antibody responses is a hallmark of a successful humoral immune response to vaccination. By inducing robust germinal centre reactions, SARS-CoV-2 mRNA-based vaccines are on track for achieving this outcome.

\section{Online content}

Any methods, additional references, Nature Research reporting summaries, source data, extended data, supplementary information, acknowledgements, peer review information; details of author contributions and competing interests; and statements of data and code availability are available at https://doi.org/10.1038/s41586-021-03738-2.

1. Mulligan, M. J. et al. Phase I/II study of COVID-19 RNA vaccine BNT162b1 in adults. Nature 586, 589-593 (2020).

2. Jackson, L. A. et al. An mRNA vaccine against SARS-CoV-2 - preliminary report. N. Engl. J. Med. 383, 1920-1931 (2020)

3. Sahin, U. et al. COVID-19 vaccine BNT162b1 elicits human antibody and $\mathrm{T}_{H} 1 \mathrm{~T}$ cell responses. Nature 586, 594-599 (2020).

4. Polack, F. P. et al. Safety and efficacy of the BNT162b2 mRNA Covid-19 vaccine. N. Engl. J. Med. 383, 2603-2615 (2020).

5. Baden, L. R. et al. Efficacy and safety of the mRNA-1273 SARS-CoV-2 vaccine. N. Engl. J. Med. 384, 403-416 (2021).

6. Zhou, X. et al. Self-replicating Semliki forest virus RNA as recombinant vaccine. Vaccine 12, 1510-1514 (1994).

7. Cagigi, A. \& Loré, K. Immune responses induced by mRNA vaccination in mice, monkeys and humans. Vaccines $\mathbf{9}, 61$ (2021).

8. Karikó, K. et al. Incorporation of pseudouridine into mRNA yields superior nonimmunogenic vector with increased translational capacity and biological stability. Mol. Ther. 16, 1833-1840 (2008).

9. Schlake, T., Thess, A., Fotin-Mleczek, M. \& Kallen, K.-J. Developing mRNA-vaccine technologies. RNA Biol. 9, 1319-1330 (2012).

10. Graham, B. S., Mascola, J. R. \& Fauci, A. S. Novel vaccine technologies: essential components of an adequate response to emerging viral diseases. J. Am. Med. Assoc. 319, 1431-1432 (2018).

11. Bettini, E. \& Locci, M. SARS-CoV-2 mRNA vaccines: immunological mechanism and beyond. Vaccines $\mathbf{9}, 147$ (2021)

12. Amit, S., Regev-Yochay, G., Afek, A., Kreiss, Y. \& Leshem, E. Early rate reductions of SARS-CoV-2 infection and COVID-19 in BNT162b2 vaccine recipients. Lancet 397, 875-877 (2021).
13. Dagan, N. et al. BNT162b2 mRNA Covid-19 vaccine in a nationwide mass vaccination setting. N. Engl. J. Med. 384, 1412-1423 (2021)

14. Vasileiou, E. et al. Interim findings from first-dose mass COVID-19 vaccination roll-out and COVID-19 hospital admissions in Scotland: a national prospective cohort study. Lancet 397, 1646-1657 (2021).

15. Case, J. B. et al. Neutralizing antibody and soluble ACE2 inhibition of a replication-competent VSV-SARS-CoV-2 and a clinical isolate of SARS-CoV-2. Cell Host Microbe 28, 475-485.e5 (2020).

16. Liu, Y. et al. Neutralizing activity of BNT162b2-elicited serum. N. Engl. J. Med. 384, 1466-1468 (2021).

17. Chen, R. E. et al. Resistance of SARS-CoV-2 variants to neutralization by monoclonal and serum-derived polyclonal antibodies. Nat. Med. 27, 717-726 (2021).

18. Leung, K., Shum, M. H., Leung, G. M., Lam, T. T. \& Wu, J. T. Early transmissibility assessment of the N501Y mutant strains of SARS-CoV-2 in the United Kingdom, October to November 2020. Euro Surveill. 26, 2002106 (2021).

19. Turner, J. S. et al. Human germinal centres engage memory and naive B cells after influenza vaccination. Nature 586, 127-132 (2020).

20. Pardi, N. et al. Expression kinetics of nucleoside-modified mRNA delivered in lipid nanoparticles to mice by various routes. J. Control. Release 217, 345-351 (2015).

21. Liang, F. et al. Efficient targeting and activation of antigen-presenting cells in vivo after modified mRNA vaccine administration in rhesus macaques. Mol. Ther. 25, 2635-2647 (2017).

22. Tai, W. et al. A novel receptor-binding domain (RBD)-based mRNA vaccine against SARS-CoV-2. Cell Res. 30, 932-935 (2020).

23. Lederer, $K$. et al. SARS-CoV-2 mRNA vaccines foster potent antigen-specific germinal center responses associated with neutralizing antibody generation. Immunity $\mathbf{5 3}$ 1281-1295.e5 (2020)

24. Kaji, T. et al. Distinct cellular pathways select germline-encoded and somatically mutated antibodies into immunological memory. J. Exp. Med. 209, 2079-2097 (2012).

25. Weisel, F. J., Zuccarino-Catania, G. V., Chikina, M. \& Shlomchik, M. J. A Temporal switch in the germinal center determines differential output of memory $B$ and plasma cells. Immunity 44, 116-130 (2016).

26. Good-Jacobson, K. L. et al. Regulation of germinal center responses and B-cell memory by the chromatin modifier MOZ. Proc. Natl Acad. Sci. USA 111, 9585-9590 (2014).

27. Bachmann, M. F., Odermatt, B., Hengartner, H. \& Zinkernagel, R. M. Induction of long-lived germinal centers associated with persisting antigen after viral infection. J. Exp. Med. 183, 2259-2269 (1996).

28. Dogan, I. et al. Multiple layers of B cell memory with different effector functions. Nat. Immunol. 10, 1292-1299 (2009).

29. Rothaeusler, K. \& Baumgarth, N. B-cell fate decisions following influenza virus infection. Eur. J. Immunol. 40, 366-377 (2010).

30. Pape, K. A., Taylor, J. J., Maul, R. W., Gearhart, P. J. \& Jenkins, M. K. Different B cell populations mediate early and late memory during an endogenous immune response. Science 331, 1203-1207 (2011).

31. Turner, J. S., Benet, Z. L. \& Grigorova, I. Transiently antigen primed B cells can generate multiple subsets of memory cells. PLoS ONE 12, e0183877 (2017).

32. Havenar-Daughton, C. et al. Rapid germinal center and antibody responses in non-human primates after a single nanoparticle vaccine immunization. Cell Rep. 29, 1756-1766.e8 (2019).

33. Cirelli, K. M. et al. Slow delivery immunization enhances HIV neutralizing antibody and germinal center responses via modulation of immunodominance. Cell 177, 1153-1171.e28 (2019).

34. Qi, H., Cannons, J. L., Klauschen, F., Schwartzberg, P. L. \& Germain, R. N. SAP-controlled T-B cell interactions underlie germinal centre formation. Nature 455, 764-769 (2008).

35. Johnston, R. J. et al. Bcl6 and Blimp-1 are reciprocal and antagonistic regulators of $T$ follicular helper cell differentiation. Science 325, 1006-1010 (2009).

36. Schmitz, A. J. et al. A public vaccine-induced human antibody protects against SARS-CoV-2 and emerging variants. Preprint at https://doi.org/10.1101/2021.03.24.436864 (2021).

37. Amanat, F. et al. SARS-CoV-2 mRNA vaccination induces functionally diverse antibodies to NTD, RBD and S2. Cell https://doi.org/10.1016/j.cell.2021.06.005 (2021).

Publisher's note Springer Nature remains neutral with regard to jurisdictional claims in published maps and institutional affiliations.

(c) The Author(s), under exclusive licence to Springer Nature Limited 2021 


\section{Methods}

No statistical methods were used to predetermine sample size.

\section{Sample collection, preparation, and storage}

All studies were approved by the Institutional Review Board of Washington University in St Louis. Written consent was obtained from all participants. Forty-one healthy volunteers were enrolled, of whom 14 provided axillary lymph node samples (Extended Data Table 1). In 5 of the 14 participants, a second draining lymph node was identified and sampled following secondary immunization. One participant (15) received the second immunization in the contralateral arm; draining lymph nodes were identified and sampled on both sides. Blood samples were collected in EDTA tubes, and PBMCs were enriched by density gradient centrifugation over Ficoll 1077 (GE) or Lymphopure (BioLegend). The residual red blood cells were lysed with ammonium chloride lysis buffer, and cells were immediately used or cryopreserved in $10 \%$ dimethylsulfoxide in fetal bovine serum (FBS). Ultrasound-guided FNA of axillary lymph nodes was performed by a radiologist or a qualified physician's assistant under the supervision of a radiologist. Lymph node dimensions and cortical thickness were measured, and the presence and degree of cortical vascularity and location of the lymph node relative to the axillary vein were determined before each FNA. For each FNA sample, six passes were made under continuous real-time ultrasound guidance using 25-gauge needles, each of which was flushed with $3 \mathrm{ml}$ of RPMI 1640 supplemented with $10 \% \mathrm{FBS}$ and $100 \mathrm{U} \mathrm{ml}^{-1}$ penicillin-streptomycin, followed by three 1-ml rinses. Red blood cells were lysed with ammonium chloride buffer (Lonza), washed with phosphate-buffered saline (PBS) supplemented with $2 \%$ FBS and $2 \mathrm{mM}$ EDTA, and immediately used or cryopreserved in $10 \%$ dimethylsulfoxide in FBS. Participants reported no adverse effects from phlebotomies or serial FNAs.

\section{Cell lines}

Expi293F cells were cultured in Expi293 Expression Medium (Gibco). Vero E6 (CRL-1586, American Type Culture Collection), Vero cells expressing TMPRSS2 (Vero-TMPRSS2 cells) ${ }^{38}$ (a gift from S. Ding), and Vero cells expressing human ACE2 and TMPRSS2 (Vero-hACE2-TMPRSS2) (a gift of A. Creanga and B. Graham) cells were cultured at $37^{\circ} \mathrm{C}$ in Dulbecco's modified Eagle medium (DMEM) supplemented with $10 \% \mathrm{FBS}, 10 \mathrm{mM}$ HEPES ( $\mathrm{pH} 7.3$ ), 1 mM sodium pyruvate, $1 \times$ nonessential amino acids and $100 \mathrm{U} \mathrm{ml}^{-1}$ of penicillin-streptomycin. Vero-TMPRSS 2 cell cultures were supplemented with $5 \mu \mathrm{g} \mathrm{ml}^{-1}$ of blasticidin. Vero-hACE2-TMPRSS2 cell cultures were supplemented with $10 \mu \mathrm{g} \mathrm{ml}^{-1}$ of puromycin.

\section{Viruses}

The 2019n-CoV/USA_WA1/2020 isolate of SARS-CoV-2 was obtained from the US Centers for Disease Control. The B.1.1.7 isolate from the UK was obtained from an infected individual. The point mutation D614G in the $S$ gene was introduced into an infectious complementary DNA clone of the $2019 n-C o V / U S A \_W A 1 / 2020$ strain as previously described $^{39}$. Nucleotide substitutions were introduced into a subclone puc57-CoV-2-F5-7 containing the $S$ gene of the SARS-CoV-2 wild-type infectious clone ${ }^{40}$.The $S$ gene of the B.1.351 variant (first identified in South Africa) was produced synthetically by Gibson assembly. The full-length infectious cDNA clones of the variant SARS-CoV-2 viruses were assembled by in vitro ligation of seven contiguous cDNA fragments following a previously described protocol ${ }^{40}$. In vitro transcription was then performed to synthesize full-length genomic RNA. To recover the mutant viruses, the RNA transcripts were electroporated into Vero E6 cells. The viruses from the supernatant of cells were collected $40 \mathrm{~h}$ later and served as $\mathrm{p} 0$ stocks. All viruses were passaged once in Vero-hACE2-TMPRSS2 cells and subjected to deep sequencing after RNA extraction to confirm the introduction and stability of substitutions ${ }^{17}$. All virus preparation and experiments were performed in an approved biosafety level 3 facility.

\section{Antigens}

Recombinant soluble SARS-CoV-2 S protein, recombinant RBD of S, human coronavirus OC43 S, and human coronavirus HKU1 S were expressed as previously described ${ }^{41}$. In brief, mammalian cell codon-optimized nucleotide sequences coding for the soluble ectodomain of the S protein of SARS-CoV-2 (GenBank: MN908947.3, amino acids 1-1213) including a C-terminal thrombin cleavage site, T4 foldon trimerization domain and hexahistidine tag, and for the RBD (amino acids 319-541) along with the signal peptide (amino acids 1-14) plus a hexahistidine tag were cloned into mammalian expression vector pCAGGS. The $S$ protein sequence was modified to remove the polybasic cleavage site (RRAR to $A$ ), and two pre-fusion stabilizing proline mutations were introduced (K986P and V987P, wild-type numbering). Expression plasmids encoding for the $\mathrm{S}$ of common human coronaviruses OC43 and HKU1 were provided by B. Graham ${ }^{42}$. Recombinant proteins were produced in Expi293F cells (ThermoFisher) by transfection with purified DNA using the ExpiFectamine 293 Transfection Kit (ThermoFisher). Supernatants from transfected cells were collected 3 days after transfection, and recombinant proteins were purified using Ni-NTA agarose (ThermoFisher), then buffer-exchanged into PBS and concentrated using Amicon Ultracel centrifugal filters (EMD Millipore). For flow cytometry staining, recombinant $\mathrm{S}$ was labelled with Alexa Fluor 647-NHS ester or biotinylated using the EZ-Link Micro NHS-PEG4-Biotinylation Kit (Thermo Fisher); excess Alexa Fluor 647 and biotin were removed using 7-kDa Zeba desalting columns (Pierce).

\section{ELISpot assay}

Plates were coated with Flucelvax Quadrivalent 2019/2020 seasonal influenza virus vaccine (Sequiris), S or RBD. A direct ex vivo ELISpot assay was performed to determine the number of total, vaccine-binding or recombinant S-binding IgG-and IgA-secreting cells present in PBMC samples using IgG/IgA double-colour ELISpot Kits (Cellular Technology) according to the manufacturer's instructions. ELISpot plates were analysed using an ELISpot counter (Cellular Technology).

\section{ELISAs}

Assays were performed in 96-well plates (MaxiSorp; Thermo) coated with $100 \mu \mathrm{l}$ of recombinant S, RBD, N-terminal domain of S (SinoBiological), OC43 S, HKU1S or bovine serum albumin diluted to $1 \mu \mathrm{g} \mathrm{ml}^{-1}$ in PBS, and plates were incubated at $4{ }^{\circ} \mathrm{C}$ overnight. Plates then were blocked with $10 \%$ FBS and $0.05 \%$ Tween 20 in PBS. Plasma or purified monoclonal antibodies were serially diluted in blocking buffer and added to the plates. Plates were incubated for $90 \mathrm{~min}$ at room temperature and then washed 3 times with $0.05 \%$ Tween 20 in PBS. Goat anti-human IgG-HRP (goat polyclonal, Jackson ImmunoResearch, 1:2,500), IgA (goat polyclonal, Jackson ImmuoResearch, 1:2,500) or IgM (goat polyclonal, Caltag, 1:4,000) were diluted in blocking buffer before adding to wells and incubating for $60 \mathrm{~min}$ at room temperature. Plates were washed 3 times with 0.05\% Tween 20 in PBS and 3 times with PBS before the addition of $o$-phenylenediamine dihydrochloride peroxidase substrate (Sigma-Aldrich). Reactions were stopped by the addition of $1 \mathrm{M}$ hydrochloric acid. Optical density measurements were taken at $490 \mathrm{~nm}$. The area under the curve for each monoclonal antibody and half-maximal binding dilution for each plasma sample were calculated using Graphpad Prism v.8.

\section{Focus reduction neutralization test}

Plasma samples were declotted by diluting 1:10 in DMEM supplemented with $2 \% \mathrm{FBS}, 10 \mathrm{mM} \mathrm{HEPES}$ and $100 \mathrm{U} \mathrm{ml}^{-1}$ penicillin-streptomycin and incubating for $3 \mathrm{~h}$ at $37^{\circ} \mathrm{C}$. Serial dilutions of resulting serum were incubated with $10^{2}$ focus-forming units of different strains or variants of SARS-CoV- 2 for $1 \mathrm{~h}$ at $37^{\circ} \mathrm{C}$. Antibody-virus complexes were added 
to Vero-TMPRSS2 cell monolayers in 96-well plates and incubated at $37^{\circ} \mathrm{C}$ for $1 \mathrm{~h}$. Subsequently, cells were overlaid with $1 \%(\mathrm{w} / \mathrm{v})$ methylcellulose in MEM supplemented with $2 \% \mathrm{FBS}$. Plates were collected $30 \mathrm{~h}$ later by removing overlays and fixed with 4\% PFA in PBS for 20 min at room temperature. Plates were washed and sequentially incubated with an oligoclonal pool of mouse anti-S monoclonal antibodies (SARS2-2, SARS2-11, SARS2-16, SARS2-31, SARS2-38, SARS2-57 and SARS2-71) (ref. ${ }^{43}$ ) and HRP-conjugated goat anti-mouse IgG (polyclonal, Sigma, 1:500) in PBS supplemented with $0.1 \%$ saponin and $0.1 \%$ bovine serum albumin. SARS-CoV-2-infected cell foci were visualized using TrueBlue peroxidase substrate (KPL) and quantified on an ImmunoSpot microanalyser (Cellular Technology).

\section{Flow cytometry and cell sorting}

Staining for flow cytometry analysis and sorting was performed using freshly isolated or cryo-preserved FNA, PBMC or tonsil samples. For analysis, cells were incubated for $30 \mathrm{~min}$ on ice with biotinylated and Alexa Fluor 647 conjugated recombinant soluble S and PD-1-BB515 (EH12.1, BD Horizon, 1:100) in 2\% FBS and 2 mM EDTA in PBS (P2), washed twice, then stained for 30 min on ice with IgG-BV480 (goat polyclonal, Jackson ImmunoResearch, 1:100), IgA-FITC (M24A, Millipore, 1:500), CD45-A532 (HI30, Thermo, 1:50), CD38-BB700 (HIT2, BD Horizon, 1:500), CD20-Pacific Blue (2H7, 1:400), CD27-BV510 (O323, 1:50), CD8-BV570 (RPA-T8, 1:200), IgM-BV605 (MHM-88, 1:100), HLA-DRBV650 (L243, 1:100), CD19-BV750 (HIB19, 1:100), CXCR5-PE-Dazzle 594 (J252D4, 1:50), IgD-PE-Cy5 (IA6-2, 1:200), CD14-PerCP(HCD14, 1:50), CD71-PE-Cy7 (CY1G4, 1:400), CD4-Spark685 (SK3, 1:200), streptavidin-APC-Fire750, CD3-APC-Fire810 (SK7, 1:50) and Zombie NIR (all BioLegend) diluted in Brilliant Staining buffer (BD Horizon). Cells were washed twice with $\mathrm{P} 2$, fixed for $1 \mathrm{~h}$ at $25^{\circ} \mathrm{C}$ using the True Nuclear fixation kit (BioLegend), washed twice with True Nuclear Permeabilization/Wash buffer, stained with FOXP3-BV421 (206D, BioLegend, 1:15), Ki-67-BV711 (Ki-67, BioLegend,1:200), Tbet-BV785 (4B10, BioLegend, 1:400), BCL6-PE (K112-91, BD Pharmingen, 1:25), and BLIMP1-A700 (646702, R\&D, 1:50) for $1 \mathrm{~h}$ at $25^{\circ} \mathrm{C}$, washed twice with True Nuclear Permeabilization/Wash buffer, and acquired on an Aurora using SpectroFlo v.2.2 (Cytek). Flow cytometry data were analysed using FlowJo v.10 (Treestar).

For sorting germinal centre B cells, FNA single-cell suspensions were stained for 30 min on ice with CD19-BV421 (HIB19, 1:100), CD3-FITC (HIT3a, 1:200), IgD-PerCP-Cy5.5 (IA6-2, 1:200), CD71-PE (CY1G4, 1:400), CXCR5-PE-Dazzle 594 (J252D4, 1:50), CD38-PE-Cy7 (HIT2, 1:200), CD20-APC-Fire750 (2H7,1:100), Zombie Aqua (all BioLegend), and Alexa Fluor 647 conjugated recombinant soluble S. For sorting plasmablasts, PBMCs were stained for 30 min on ice with CD20-PB $(2 \mathrm{H} 7$, 1:400), CD71-FITC (CY1G4, 1:200), CD4-PerCP (OKT4, 1:100), IgD-PE (IA6-2, 1:200), CD38-PE-Cy7 (HIT2, 1:200), CD19-APC (HIB19, 1:200) and Zombie Aqua (all BioLegend). Cells were washed twice, and single $S$-binding germinal centre $B$ cells (live singlet $C D 3^{-} \mathrm{CD} 19^{+} \mathrm{IgD}^{\text {low }} \mathrm{CD} 20^{\text {high }}$ $\mathrm{CD} 38^{\text {int }} \mathrm{CD}{ }^{+} \mathrm{CXCR}^{+} \mathrm{S}^{+}$) were sorted using a FACSAria II into 96-well plates containing $2 \mu \mathrm{l}$ Lysis Buffer (Clontech) supplemented with $1 \mathrm{U} \mu^{-1}$ RNase inhibitor (NEB), or total germinal centre B cells or plasmablasts (live singlet $\mathrm{CD}^{-} \mathrm{CD} 19^{+} \mathrm{IgD}^{\text {low }} \mathrm{CD} 2 \mathrm{O}^{\text {low }} \mathrm{CD} 38^{+} \mathrm{CD} 71^{+}$) were bulk-sorted into buffer RLT Plus (Qiagen) and immediately frozen on dry ice.

\section{Monoclonal antibody generation}

Antibodies were cloned as previously described ${ }^{44}$. In brief, VH, VK and $\mathrm{V} \lambda$ genes were amplified by reverse transcription PCR and nested $P C R$ reactions from singly sorted germinal centre $B$ cells using primer combinations specific for IgG, IgM, IgA, IgK and Ig $\lambda$ from previously described primer sets ${ }^{45}$, and then sequenced. To generate recombinant antibodies, restriction sites were incorporated via PCR with primers to the corresponding heavy and light chain $\mathrm{V}$ and $\mathrm{J}$ genes. The amplified $\mathrm{VH}, \mathrm{VK}$ and $\mathrm{V} \lambda$ genes were cloned into IgG1 and IgK or Ig $\lambda$ expression vectors, respectively, as previously described ${ }^{45-47}$. Heavy and light chain plasmids were co-transfected into Expi293F cells (Gibco) for expression, and antibody was purified using protein A agarose chromatography (Goldbio). Sequences were obtained from PCR reaction products and annotated using the ImMunoGeneTics (IMGT)/V-QUEST database (http://www.imgt.org/IMGT_vquest/) ${ }^{48,49}$. Mutation frequency was calculated by counting the number of nonsynonymous nucleotide mismatches from the germline sequence in the heavy chain variable segment leading up to the CDR3, while excluding the $5^{\prime}$ primer sequences that could be error-prone.

\section{Bulk B cell receptor sequencing}

RNA was purified from sorted plasmablasts from PBMCs and germinal centre B cells from lymph nodes from participants 07, 20 (lymph node 1) and 22 using the RNeasy Plus Micro kit (Qiagen). Reverse transcription, unique molecular identifier (UMI) barcoding, cDNA amplification, and Illumina linker addition to B cell heavy chain transcripts were performed using the human NEBNext Immune Sequencing Kit (New England Biolabs) according to the manufacturer's instructions. High-throughput $2 \times 300$-bp paired-end sequencing was performed on the Illumina MiSeq platform with a $30 \%$ PhiX spike-in according to manufacturer's recommendations, except for performing 325 cycles for read 1 and 275 cycles for read 2 .

\section{Processing of $B$ cell receptor bulk-sequencing reads}

Demultiplexed pair-end reads were BLAST'ed using blastn v.2.11.0 (ref..$^{50}$ ) for PhiX removal and subsequently preprocessed using pRESTO v.0.6.2 (ref. ${ }^{51}$ ) as follows. (1) Reads with a mean Phred quality score below 20 were filtered. (2) Reads were aligned against template switch sequences and constant region primers (Extended Data Table 5), with a maximum mismatch rate of 0.5 and 0.2 respectively. (3) A UMI was assigned to each read by extracting the first 17 nucleotides preceding the template switch site. (4) Sequencing and multiplexing errors in the UMI region were then corrected using a previously published approach ${ }^{52}$. In brief, reads with similar UMIs were clustered using cd-hit-est v.4.8.1 (ref. ${ }^{53}$ ) on the basis of the pairwise distance of their UMIs with a similarity threshold of 0.83 that was estimated from 10,000 reads. The UMI-based read groups were further clustered within themselves on the basis of the pairwise distance of the non-UMI region of their reads with a similarity threshold of 0.8 . Read clusters spanning multiple multiplexed samples were assigned to the majority sample. (5) Separate consensus sequences for the forward and reverse reads within each read cluster were constructed with a maximum error score of 0.1 and minimum constant region primer frequency of 0.6 . If multiple constant region primers were associated with a particular read cluster, the majority primer was used. (6) Forward and reverse consensus sequence pairs were assembled by first attempting de novo assembly with a minimum overlap of 8 nucleotides and a maximum mismatch rate of 0.3 . If unsuccessful, this was followed by reference-guided assembly using blastn v.2.11.0 (ref. ${ }^{50}$ ) with a minimum identity of 0.5 and an $E$-value threshold of $1 \times 10^{-5}$. (7) Isotypes were assigned by local alignment of the $3^{\prime}$ end of each consensus sequence to isotype-specific internal constant region sequences (Extended Data Table 5) with a maximum mismatch rate of 0.3 . Sequences with inconsistent isotype assignment and constant region primer alignment were removed. (8) Duplicate consensus sequences, except those with different isotype assignments, were collapsed into unique sequences. Only unique consensus sequences with at least two contributing reads were used subsequently (Extended Data Table 6).

\section{$B$ cell receptor genotyping}

Initial germline $\mathrm{V}(\mathrm{D}) \mathrm{J}$ gene annotation was performed using IgBLAST v.1.17.1 (ref. ${ }^{54}$ ) with IMGT/GENE-DB release 202113-2 (ref. ${ }^{55}$ ). IgBLAST output was parsed using Change-O v.1.0.2 (ref. ${ }^{56}$ ). Quality control was performed, requiring each sequence to have non-empty $\mathrm{V}$ and $\mathrm{J}$ gene annotations; exhibit chain consistency in all annotations; bear fewer 
than 10 non-informative (non-A/T/G/C, such as N or -) positions; and carry a CDR3 with no $\mathrm{N}$ and a nucleotide length that is a multiple of 3. Individualized genotypes were inferred using TIgGER v.1.0.0 (ref. ${ }^{57}$ ) and used to finalize V(D)J annotations. Sequences annotated as non-productively rearranged by IgBLAST were removed from further analysis.

\section{Clonal lineage analysis}

B cell clonal lineages were inferred on the basis of productively rearranged heavy chain sequences using hierarchical clustering with single linkage ${ }^{58,59}$.Sequences were first partitioned based on common $V$ and J gene annotations and CDR3 lengths. Within each partition, sequences with CDR3s that were within 0.15 normalized Hamming distance from each other were clustered as clones. This distance threshold was determined by manual inspection in conjunction with kernel density estimates to identify the local minimum between the two modes of the within-participant bimodal distance-to-nearest distribution (Extended Data Fig. 3a). Following clonal clustering, full-length clonal consensus germline sequences were reconstructed for each clone with D-segment and N/P regions masked with Ns, resolving any ambiguous gene assignments by majority rule. Within each clone, duplicate IMGT-aligned V(D) J sequences from bulk sequencing were collapsed with the exception of duplicates derived from different $B$ cell compartments or isotypes. Clones were visualized as networks ${ }^{60}$ using igraph v.1.2.5 (ref. ${ }^{61}$ ). First, a full network was calculated for each clone, in which an edge was drawn between every pair of sequences with CDR3s that were within 0.15 normalized Hamming distance from each other. Then, a minimum spanning tree was derived from the full network, in which only edges essential for ensuring that all sequences connected in the full network remain connected in the minimum spanning tree either directly or indirectly were retained. The minimum spanning tree was then visualized for each clone.

\section{Calculation of somatic hypermutation frequency}

Mutation frequency was calculated by counting the number of nucleotide mismatches from the germline sequence in the observed heavy chain variable segment leading up to the CDR3, while excluding the first 18 positions that could be error-prone owing to the primers used for generating the monoclonal antibody sequences. Calculation was performed using the calcObservedMutations function from SHazaM v.1.0.2 $\left(\right.$ ref. $^{56}$ ).

\section{Reporting summary}

Further information on research design is available in the Nature Research Reporting Summary linked to this paper.

\section{Data availability}

Antibody sequences are deposited on GenBank under the following accession numbers: MW926396-MW926407, MW926409-MW926430, MW926432-MW926441 and MZ292481-MZ292510, available from GenBank/EMBL/DDBJ. Bulk sequencing reads are deposited on Sequence Read Archive under BioProject PRJNA731610. Processed B cell receptor data are deposited at https://doi.org/10.5281/zenodo.5042252. The IMGT/V-QUEST database is accessible at http://www.imgt.org/IMGT_ vquest/. Other relevant data are available from the corresponding authors upon request.

38. Zang, R. et al. TMPRSS 2 and TMPRSS4 promote SARS-CoV-2 infection of human small intestinal enterocytes. Sci. Immunol. 5, eabc3582 (2020)

39. Plante, J. A. et al. Spike mutation D614G alters SARS-CoV-2 fitness. Nature 592, 116-121 (2021).

40. Xie, X. et al. An infectious cDNA clone of SARS-CoV-2. Cell Host Microbe 27, 841-848.e3 (2020).

41. Stadlbauer, D. et al. SARS-CoV-2 seroconversion in humans: a detailed protocol for a serological assay, antigen production, and test setup. Curr. Protoc. Microbiol. 57, e100 (2020).
42. Pallesen, J. et al. Immunogenicity and structures of a rationally designed prefusion MERS-CoV spike antigen. Proc. Natl Acad. Sci. USA 114, E7348-E7357 (2017).

43. Liu, Z. et al. Identification of SARS-CoV-2 spike mutations that attenuate monoclonal and serum antibody neutralization. Cell Host Microbe 29, 477-488.e4 (2021).

44. Wrammert, J. et al. Broadly cross-reactive antibodies dominate the human B cell response against 2009 pandemic H1N1 influenza virus infection. J. Exp. Med. 208, 181-193 (2011).

45. Smith, K. et al. Rapid generation of fully human monoclonal antibodies specific to a vaccinating antigen. Nat. Protoc. 4, 372-384 (2009).

46. Wrammert, J. et al. Rapid cloning of high-affinity human monoclonal antibodies against influenza virus. Nature 453, 667-671 (2008).

47. Nachbagauer, R. et al. broadly reactive human monoclonal antibodies elicited following pandemic $\mathrm{H} 1 \mathrm{~N} 1$ influenza virus exposure protect mice against highly pathogenic $\mathrm{H} 5 \mathrm{~N} 1$ challenge. J. Virol. 92, 1-17 (2018).

48. Brochet, X., Lefranc, M.-P. \& Giudicelli, V. IMGT/V-QUEST: the highly customized and integrated system for IG and TR standardized V-J and V-D-J sequence analysis. Nucleic Acids Res. 36, W503-W508 (2008)

49. Giudicelli, V., Brochet, X. \& Lefranc, M.-P. IMGT/V-QUEST: IMGT standardized analysis of the immunoglobulin (IG) and T cell receptor (TR) nucleotide sequences. Cold Spring Harb. Protoc. 2011, pdb.prot5633 (2011).

50. Camacho, C. et al. BLAST+: architecture and applications. BMC Bioinformatics 10, 421 (2009).

51. Vander Heiden, J. A. et al. pRESTO: a toolkit for processing high-throughput sequencing raw reads of lymphocyte receptor repertoires. Bioinformatics 30, 1930-1932 (2014)

52. Jiang, R. et al. Thymus-derived $B$ cell clones persist in the circulation after thymectomy in myasthenia gravis. Proc. Natl Acad. Sci. USA 117, 30649-30660 (2020).

53. Li, W. \& Godzik, A. Cd-hit: a fast program for clustering and comparing large sets of protein or nucleotide sequences. Bioinformatics 22, 1658-1659 (2006).

54. Ye, J., Ma, N., Madden, T. L. \& Ostell, J. M. IgBLAST: an immunoglobulin variable domain sequence analysis tool. Nucleic Acids Res. 41, W34-W4O (2013).

55. Giudicelli, V., Chaume, D. \& Lefranc, M. P. IMGT/GENE-DB: a comprehensive database for human and mouse immunoglobulin and T cell receptor genes. Nucleic Acids Res. 33, D256-D261 (2005)

56. Gupta, N. T. et al. Change-O: a toolkit for analyzing large-scale B cell immunoglobulin repertoire sequencing data. Bioinformatics 31, 3356-3358 (2015).

57. Gadala-Maria, D., Yaari, G., Uduman, M. \& Kleinstein, S. H. Automated analysis of high-throughput B-cell sequencing data reveals a high frequency of novel immunoglobulin V gene segment alleles. Proc. Natl Acad. Sci. USA 112, E862-E870 (2015).

58. Gupta, N. T. et al. Hierarchical clustering can identify B cell clones with high confidence in Ig repertoire sequencing data. J. Immunol. 198, 2489-2499 (2017).

59. Zhou, J. Q. \& Kleinstein, S. H. Cutting edge: Ig $\mathrm{H}$ chains are sufficient to determine most $\mathrm{B}$ cell clonal relationships. J. Immunol. 203, 1687-1692 (2019).

60. Bashford-Rogers, R. J. M. et al. Network properties derived from deep sequencing of human B-cell receptor repertoires delineate B-cell populations. Genome Res. 23 1874-1884 (2013).

61. Csardi, G. \& Nepusz, T. The igraph software package for complex network research. InterJournal Complex Syst. 1695, 1-9 (2006).

Acknowledgements We thank the donors for providing specimens, L. Kessels, A. J. Winingham, K. Safi and the staff of the Infectious Diseases Clinical Research Unit at Washington University School of Medicine for assistance with scheduling participants and sample collection, and E. Lantelme for facilitating sorting. The Vero-TMPRSS2 cells were provided by S. Ding. The expression plasmids encoding the S of coronaviruses OC43 and HKU1 were provided by K. Corbett and B. Graham. The laboratory of A.H.E. was supported by National Institute of Allergy and Infectious Diseases (NIAID) grants U01Al141990 and 1U01AI150747 and NIAID Centers of Excellence for Influenza Research and Surveillance (CEIRS) contract HHSN272201400006C. The laboratories of A.H.E. and F.K. were supported by NIAID CEIRS contract HHSN272201400008C and NIAID Collaborative Influenza Vaccine Innovation Centers contract 75N93019C00051. The laboratory of M.S.D. was supported by R01 Al157155. The laboratory of P.Y.-S. was supported by NIH grants Al134907 and UL1TR001439, and awards from the Sealy \& Smith Foundation, Kleberg Foundation, the John S. Dunn Foundation, the Amon G. Carter Foundation, the Gilson Longenbaugh Foundation and the Summerfield Robert Foundation. J.S.T. was supported by NIAID 5T32CA009547. J.B.C. was supported by a Helen Hay Whitney Foundation postdoctoral fellowship. The WU368 study was reviewed and approved by the Washington University Institutional Review Board (approval no. 202012081).

Author contributions A.H.E., J.A.O. and R.M.P. conceived and designed the study. J.A.O., A.H., M.K.K. and R.M.P. wrote and maintained the Institutional Review Board protocol, recruited and phlebotomized participants, and coordinated sample collection. J.S.T., E.K., W.K., A.J.S. and T.L. processed specimens. J.S.T., E.K., W.K. and A.J.S. performed ELISA and ELISpot. R.E.C. and J.B.C. performed neutralization assays. J.S.T., E.K., W.K., A.J.S., T.L. and M.T. generated and characterized monoclonal antibodies. A.J.S. performed RNA extractions and library preparation for B cell receptor bulk sequencing. J.Q.Z. analysed B cell receptor repertoire data. T.S. and W.D.M. performed FNA. W.D.M. and S.A.T. supervised lymph node evaluation prior to FNA and specimen collection and evaluated lymph node ultrasound data. A.J.S. expressed SARS-CoV-2 S and RBD proteins. F.A. and F.K. expressed coronavirus S proteins. J.S.T. sorted cells and collected and analysed the flow cytometry data. X.X. and P.-Y.S. prepared the SARS-CoV-2 with variant S mutations. J.S.T., A.M.R. and A.H.E. analysed the data. M.S.D. and A.H.E. supervised experiments and obtained funding. J.S.T. and A.H.E. composed the manuscript. All authors reviewed the manuscript.

Competing interests The laboratory of A.H.E. received funding under sponsored research agreements that are unrelated to the data presented in the current study from Emergent BioSolutions and from AbbVie. A.H.E. is a consultant for Mubadala Investment Company and the founder of ImmuneBio Consulting. J.S.T., A.J.S., M.S.D. and A.H.E. are recipients of a 
licensing agreement with Abbvie that is unrelated to the data presented in the current study. M.S.D. is a consultant for Inbios, Vir Biotechnology, Fortress Biotech and Carnival Corporation, and on the Scientific Advisory Boards of Moderna and Immunome. The laboratory of M.S.D. has received unrelated funding support in sponsored research agreements from Moderna, Vir Biotechnology and Emergent BioSolutions. A patent application related to this work has been filed by Washington University School of Medicine. The Icahn School of Medicine at Mount Sinai has filed patent applications relating to SARS-CoV-2 serological assays and NDV-based SARS-CoV-2 vaccines which list F.K. as co-inventor. Mount Sinai has spun out a company, Kantaro, to market serological tests for SARS-CoV-2. F.K. has consulted for Merck and Pfizer (before 2020), and is currently consulting for Pfizer, Seqirus and Avimex. The laboratory of F.K. is also collaborating with Pfizer on animal models of SARS-CoV-2. The laboratory of P.-Y.S. has received sponsored research agreements from Pfizer, Gilead, Merck and IGM Sciences Inc. The content of this manuscript is solely the responsibility of the authors and does not necessarily represent the official view of NIAID or NIH.

Additional information

Supplementary information The online version contains supplementary material available at https://doi.org/10.1038/s41586-021-03738-2.

Correspondence and requests for materials should be addressed to R.M.P. or A.H.E.

Peer review information Nature thanks the anonymous reviewers for their contribution to the peer review of this work. Peer reviewer reports are available.

Reprints and permissions information is available at http://www.nature.com/reprints. 


\section{Article}

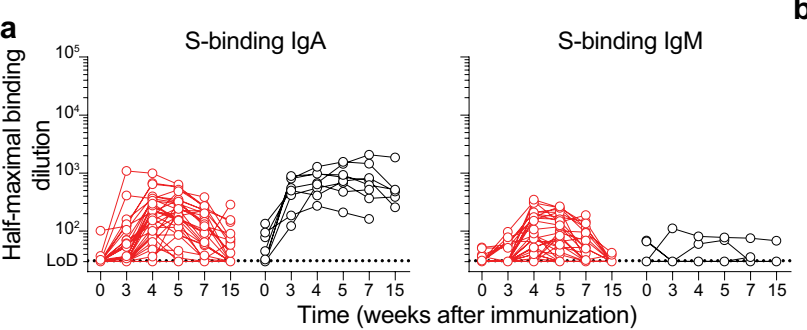

Extended Data Fig. 1 | Antibody response to SARS-CoV-2 immunization. a, Plasma IgA (left) and IgM (right) titres against SARS-CoV-2 S measured by ELISA in participants without (red) and with (black) a history of SARS-CoV-2 infection at baseline, and 3, 4, 5, 7 and 15 weeks after immunization. b, Neutralizing activity of serum against WA1/2020 D614G (left), B.1.1.7 (middle) and a chimeric virus expressing B.1.351S (right) in Vero-TMPRSS2 cells at

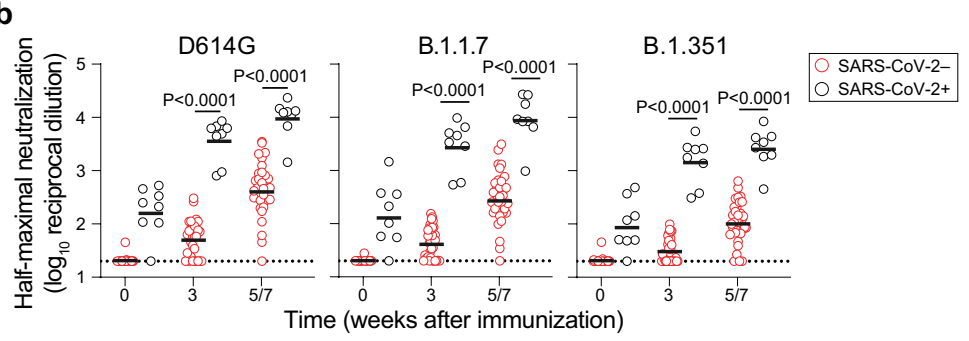

baseline, 3, and 5 or 7 weeks after immunization in participants without (red) and with (black) a history of SARS-CoV-2 infection. $P$ values from two-sided Mann-Whitney tests. Dotted lines indicate limits of detection. Horizontal lines indicate the geometric mean. Symbols at each time point represent one sample $(n=41)$. Results are from one experiment performed in duplicate. 

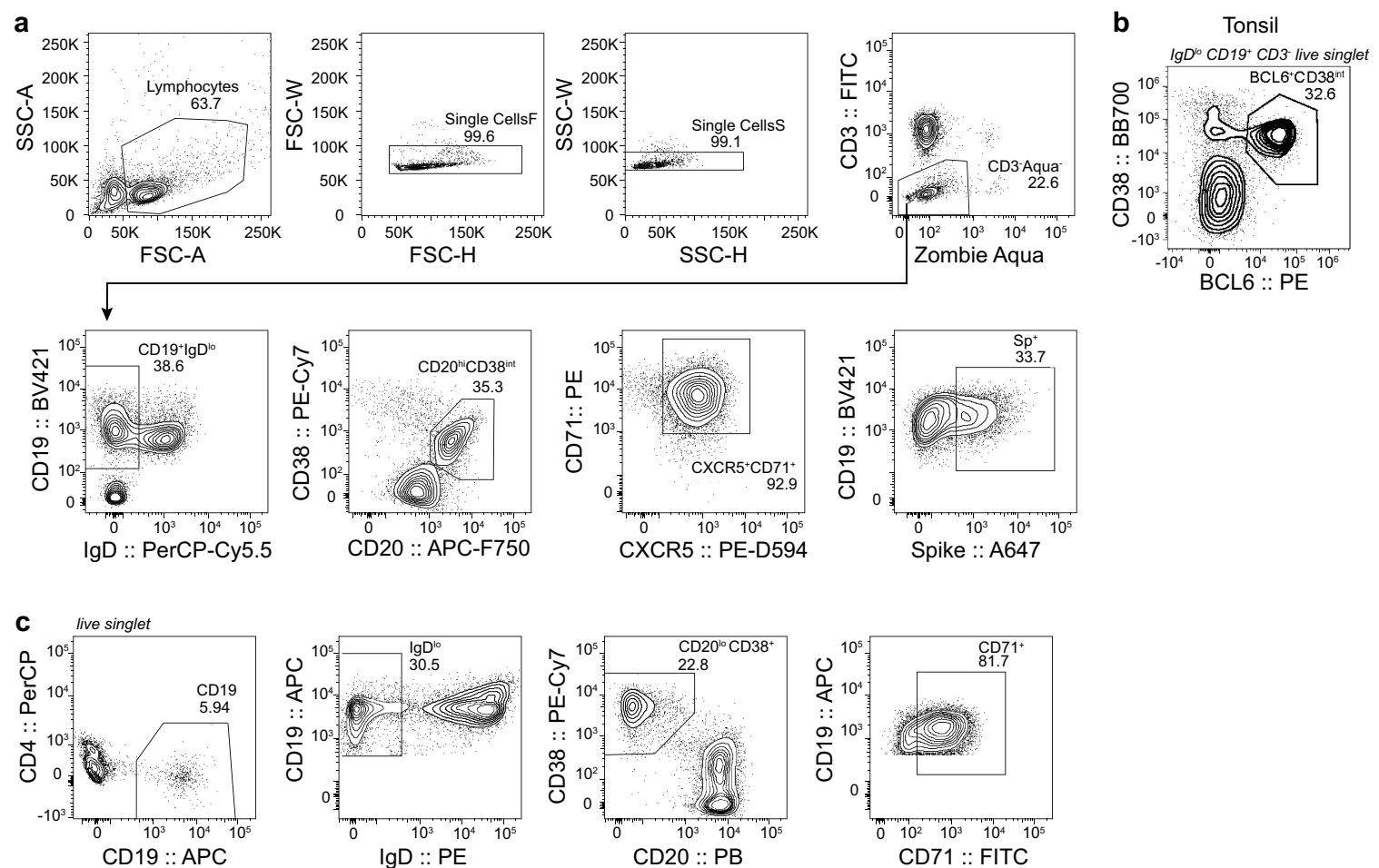

Extended Data Fig. 2 | Gating strategies for analysis of germinal centre response to SARS-CoV-2 immunization. a, c, Sorting gating strategies for S-binding germinal centre B cells from FNAs (a) and total plasmablasts from PBMCs (c). b. Representative plot of germinal centre B cells in tonsil. 


\section{Article}
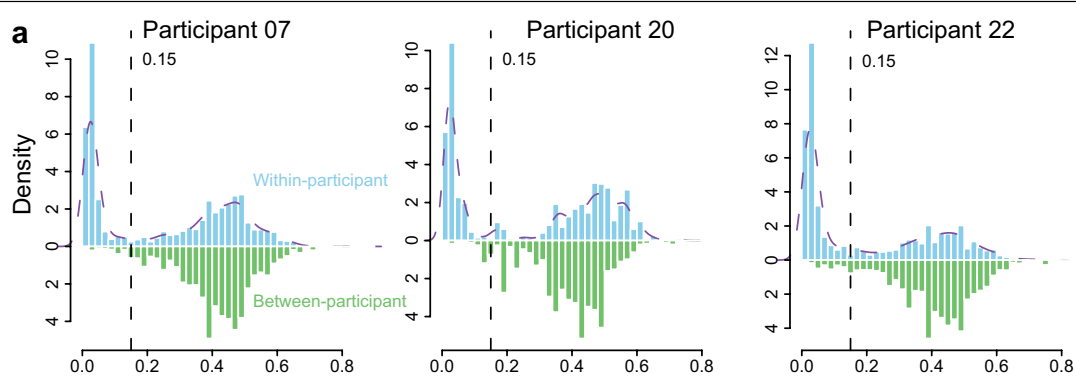

b

Distance to Nearest Neighbor

Participant 20

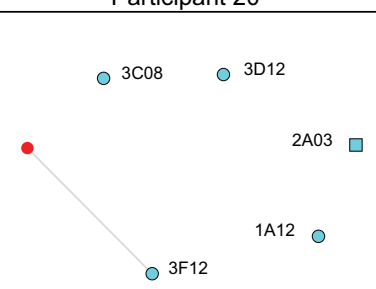

Extended Data Fig. 3 | Clonal analysis of germinal centre response to SARS-CoV-2 immunization. a, Distance-to-nearest-neighbour plots for choosing a distance threshold for inferring clones via hierarchical clustering. After partitioning sequences based on common $V$ and J genes and CDR3 length, the nucleotide Hamming distance of a CDR3 to its nearest nonidentical neighbour from the same participant within its partition was calculated and normalized by CDR3 length (blue histogram). For reference, the distance to the nearest nonidentical neighbour from other participants was calculated (green histogram). A clustering threshold of 0.15 (dashed black line) was chosen via manual inspection and kernel density estimate (dashed purple line) to separate the two modes of the within-participant distance distribution representing, respectively, sequences that were probably clonally related and unrelated. b, Clonal relationship of sequences from S-binding germinal centre-derived monoclonal antibodies (cyan) to sequences from bulk repertoire analysis of plasmablasts sorted from PBMCs (red) and germinal centre B cells (blue) 4 weeks after immunization. Each clone is visualized as a network in which each node represents a sequence and sequences are linked as a minimum spanning tree of the network. Symbol shape indicates sequence isotype: IgG (circle), IgA (star) and IgM (square); symbol size corresponds to sequence count. 

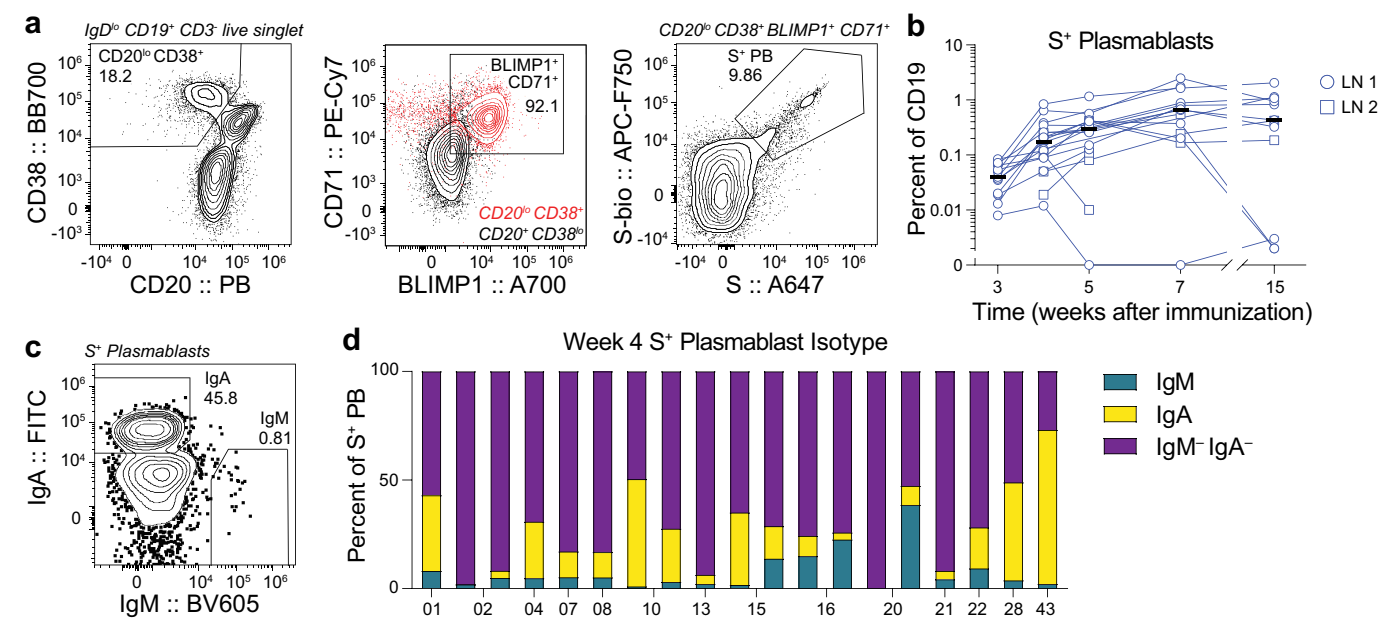

Extended Data Fig. 4 | Lymph node plasmablast response to SARS-CoV-2 immunization. a, c, Representative flow cytometry plots showing gating of $\mathrm{CD} 20^{\text {low }} \mathrm{CD} 38^{+} \mathrm{CD}^{-1} 1^{+} \mathrm{BLIMP}^{+} \mathrm{S}^{+}$plasmablasts from IgD $^{\text {low }} \mathrm{CD} 19^{+} \mathrm{CD}^{-}$live singlet lymphocytes (a) and IgA and IgM staining on $\mathrm{S}^{+}$plasmablasts (c) in FNA

nodes. Symbols at each time point represent one FNA sample; square symbols denote second lymph node sampled $(n=14)$. Horizontal lines indicate the median. d, Percentages of $\operatorname{IgM}^{+}$(teal), $\operatorname{IgA}^{+}$(yellow) or $\operatorname{IgM}^{-} \operatorname{Ig} \mathrm{A}^{-}$(purple) samples. $\mathbf{b}$, Kinetics of $\mathrm{S}^{+}$plasmablasts gated as in a from FNA of draining lymph $\mathrm{S}^{+}$plasmablasts gated as in c in FNA of draining lymph nodes 4 weeks after primary immunization. Each bar represents one sample $(n=14)$. 


\section{Article}

Extended Data Table 1 | Participant demographics

\begin{tabular}{|c|c|c|}
\hline Variable & $\begin{array}{c}\text { Total } N=41 \\
N(\%)\end{array}$ & $\begin{array}{c}\text { Lymph node } \mathrm{N}=14 \\
\mathrm{~N}(\%)\end{array}$ \\
\hline Age (median [range]) & $37(28-73)$ & $37(28-52)$ \\
\hline \multicolumn{3}{|l|}{ Sex } \\
\hline Female & $18(43.9)$ & $8(57.1)$ \\
\hline Male & $23(56.1)$ & $6(42.9)$ \\
\hline \multicolumn{3}{|l|}{ Race } \\
\hline White & $30(73.2)$ & $11(78.6)$ \\
\hline Asian & $9(22)$ & $2(14.3)$ \\
\hline Black & $1(2.4)$ & $1(7.1)$ \\
\hline Other & $1(2.4)$ & $0(0)$ \\
\hline \multicolumn{3}{|l|}{ Ethnicity } \\
\hline Not of Hispanic, Latinx, or Spanish origin & $39(95.1)$ & $13(92.9)$ \\
\hline Hispanic, Latinx, Spanish origin & $2(4.9)$ & $1(7.1)$ \\
\hline BMI (median [range]) & $25.4(21.4-40)$ & $23.9(21.4-40)$ \\
\hline \multicolumn{3}{|l|}{ Comorbidities } \\
\hline Lung disease & $2(4.9)$ & $1(7.1)$ \\
\hline Diabetes mellitus & $0(0)$ & $0(0)$ \\
\hline Hypertension & $7(17.1)$ & $2(14.3)$ \\
\hline Cardiovascular & $0(0)$ & $0(0)$ \\
\hline Liver disease & $0(0)$ & $0(0)$ \\
\hline Chronic kidney disease & $0(0)$ & $0(0)$ \\
\hline Cancer on chemotherapy & $0(0)$ & $0(0)$ \\
\hline Hematological malignancy & $0(0)$ & $0(0)$ \\
\hline Pregnancy & $0(0)$ & $0(0)$ \\
\hline Neurological & $0(0)$ & $0(0)$ \\
\hline HIV & $0(0)$ & $0(0)$ \\
\hline Solid organ transplant recipient & $0(0)$ & $0(0)$ \\
\hline Bone marrow transplant recipient & $0(0)$ & $0(0)$ \\
\hline Hyperlipidemia & $1(2.4)$ & $0(0)$ \\
\hline Confirmed SARS-CoV-2 infection & $8(19.5)$ & $0(0)$ \\
\hline $\begin{array}{l}\text { Time from SARS-CoV-2 infection to baseline visit in } \\
\text { days (median [range]) }\end{array}$ & $122(50-307)$ & -- \\
\hline
\end{tabular}


Extended Data Table 2 | Vaccine side effects

\begin{tabular}{lccc}
\hline Variable & $\begin{array}{c}\text { Total } \mathbf{N = 4 1} \\
\mathbf{N}(\%)\end{array}$ & $\begin{array}{c}\text { Total N=41 } \\
\mathbf{N}(\%)\end{array}$ \\
\hline First dose & & Second dose & $1(2.4)$ \\
None & $3(7.3)$ & None & $15(36.6)$ \\
Chills & $5(12.2)$ & Chills & $6(14.6)$ \\
Fever & $2(4.9)$ & Fever & $11(26.8)$ \\
Headache & $6(14.6)$ & Headache & $36(87.8)$ \\
Injection site pain & $33(80.5)$ & Injection site pain & $32(78)$ \\
Muscle or joint pain & $9(21.9)$ & Muscle or joint pain & $23(56.1)$ \\
Fatigue & $8(19.5)$ & Fatigue & $2(4.8)$ \\
Sweating & $0(0)$ & Sweating & \\
& & & $24(4-48)$ \\
Chills & Duration of side effects in hours (median [range]) & $24(1-48)$ \\
Fever & $48(6-72)$ & Chills & $24(4-48)$ \\
Headache & $9(6-12)$ & Fever & $36(2-120)$ \\
Injection site pain & $9(3-48)$ & Headache & $24(1-48)$ \\
Muscle or joint pain & $36(2-120)$ & Injection site pain & $24(3-144)$ \\
Fatigue & $36(1-48)$ & Muscle or joint pain & $18(18)$ \\
Sweating & $30(3-48)$ & Fatigue & \\
& $0(0)$ & Sweating & \\
\end{tabular}


Article

Extended Data Table 3 | Lymph node population frequencies

\begin{tabular}{|c|c|c|c|c|c|c|}
\hline Participant & Week post immunization & LN \# & Total GC (\%CD19) & S+ GC (\%CD19) & S+ PB (\%CD19) & CD14 (\%live singlet) \\
\hline 01 & 3 & 1 & 15.1744 & 3.2617 & 0.0718 & 0.2134 \\
\hline 01 & 4 & 1 & 13.7195 & 5.4700 & 0.8436 & 0.0588 \\
\hline 01 & 5 & 1 & 11.1280 & 4.0445 & 1.1611 & 0.1461 \\
\hline 01 & 7 & 1 & 31.1819 & 13.5866 & 1.6436 & 0.1469 \\
\hline 01 & 15 & 1 & 29.0930 & 18.1334 & 2.0446 & 0.1628 \\
\hline 02 & 3 & 1 & 8.2782 & 1.3827 & 0.0132 & 0.1590 \\
\hline 02 & 4 & 1 & 34.1504 & 9.7890 & 0.2102 & 0.1705 \\
\hline 02 & 4 & 2 & 14.0743 & 4.7121 & 0.2521 & 0.1371 \\
\hline 02 & 5 & 1 & 44.6608 & 11.6465 & 0.4086 & 0.0743 \\
\hline 02 & 5 & 2 & 13.5353 & 3.8936 & 0.2893 & 0.1361 \\
\hline 02 & 7 & 1 & 21.8959 & 6.0492 & 0.3725 & 0.2486 \\
\hline 02 & 7 & 2 & 23.1123 & 9.3883 & 0.5557 & 0.7063 \\
\hline 02 & 15 & 1 & 7.1063 & 0.0197 & 0.0020 & 0.1649 \\
\hline 04 & 3 & 1 & 4.6727 & 0.5706 & 0.0538 & 0.1934 \\
\hline 04 & 4 & 1 & 13.9308 & 2.8621 & 0.0885 & 0.3733 \\
\hline 04 & 5 & 1 & 11.3856 & 5.9721 & 0.4296 & 0.7237 \\
\hline 04 & 7 & 1 & 43.8266 & 6.4556 & 0.2019 & 0.1436 \\
\hline 04 & 15 & 1 & 3.7193 & 0.7127 & 0.0025 & 0.9901 \\
\hline 07 & 3 & 1 & 21.0403 & 4.6411 & 0.0697 & 0.1927 \\
\hline 07 & 4 & 1 & 19.6634 & 4.9771 & 0.2599 & 0.1507 \\
\hline 07 & 5 & 1 & 11.3504 & 3.8557 & 0.2576 & 0.4177 \\
\hline 07 & 7 & 1 & 39.2049 & 14.7032 & 0.6765 & 0.6582 \\
\hline 07 & 15 & 1 & 28.9957 & 20.1221 & 0.3248 & 0.0349 \\
\hline 08 & 3 & 1 & 9.9010 & 1.2181 & 0.0651 & 0.2738 \\
\hline 08 & 4 & 1 & 1.3233 & 0.4150 & 0.0904 & 0.1670 \\
\hline 08 & 5 & 1 & 3.9913 & 0.9238 & 0.1507 & 0.3377 \\
\hline 08 & 7 & 1 & 12.1411 & 4.2393 & 0.7559 & 0.8097 \\
\hline 10 & 3 & 1 & 7.7130 & 1.1146 & 0.0399 & 0.2216 \\
\hline 10 & 4 & 1 & 5.9172 & 1.4892 & 0.3494 & 0.0676 \\
\hline 10 & 4 & 2 & 5.7036 & 1.3733 & 0.1867 & 0.0860 \\
\hline 10 & 5 & 1 & 2.8213 & 0.9125 & 0.3428 & 0.0772 \\
\hline 10 & 5 & 2 & 3.4006 & 1.0486 & 0.4156 & 0.0517 \\
\hline 10 & 7 & 1 & 7.3456 & 3.1376 & 0.8776 & 0.2061 \\
\hline 10 & 7 & 2 & 4.2626 & 1.0628 & 0.1663 & 0.1314 \\
\hline 10 & 15 & 1 & 19.2991 & 14.2480 & 1.0759 & 0.0340 \\
\hline 10 & 15 & 2 & 7.0114 & 2.7615 & 0.1849 & 0.0292 \\
\hline 13 & 3 & 1 & 14.8994 & 3.5681 & 0.0199 & 0.5633 \\
\hline 13 & 4 & 1 & 8.5564 & 3.0085 & 0.2116 & 0.3352 \\
\hline 13 & 5 & 1 & 7.1981 & 2.1647 & 0.3036 & 0.4254 \\
\hline 13 & 7 & 1 & 15.9621 & 3.7382 & 0.6532 & 0.3253 \\
\hline 13 & 15 & 1 & 20.1410 & 10.1032 & 0.4304 & 1.6315 \\
\hline 15 & 3 & 1 & 13.0526 & 2.9882 & 0.0838 & 0.0715 \\
\hline 15 & 4 & 1 & 26.8834 & 8.1055 & 0.1196 & 0.1948 \\
\hline 15 & 4 & 2 & 0.3157 & 0.0169 & 0.1039 & 0.1379 \\
\hline 15 & 5 & 1 & 44.5687 & 9.7412 & 0.3016 & 0.0786 \\
\hline 15 & 5 & 2 & 0.6237 & 0.0247 & 0.0098 & 0.2388 \\
\hline 16 & 3 & 1 & 5.1983 & 0.7456 & 0.0195 & 0.2619 \\
\hline 16 & 4 & 1 & 7.6114 & 1.5173 & 0.0510 & 0.1431 \\
\hline 16 & 4 & 2 & 1.2226 & 0.2419 & 0.0190 & 0.1142 \\
\hline 16 & 5 & 1 & 17.0381 & 3.3806 & 0.1261 & 0.0570 \\
\hline 16 & 5 & 2 & 7.4397 & 1.8530 & 0.1101 & 0.1405 \\
\hline 20 & 3 & 1 & 0.4058 & 0.0257 & 0.0076 & 0.2140 \\
\hline 20 & 4 & 1 & 7.4714 & 3.0853 & 0.0115 & 0.1478 \\
\hline 20 & 4 & 2 & 7.8030 & 1.2875 & 0.0505 & 0.1149 \\
\hline 20 & 5 & 1 & 2.0994 & 0.3146 & 0.0000 & 0.2934 \\
\hline 20 & 5 & 2 & 3.3579 & 0.7320 & 0.0811 & 0.1607 \\
\hline 20 & 7 & 1 & 0.5222 & 0.0378 & 0.0000 & 0.4082 \\
\hline 20 & 7 & 2 & 13.6571 & 4.4611 & 0.2402 & 0.1100 \\
\hline 20 & 15 & 1 & 0.4687 & 0.0391 & 0.0033 & 0.8360 \\
\hline 20 & 15 & 2 & 16.9158 & 11.1431 & 0.4222 & 0.2930 \\
\hline 21 & 4 & 1 & 20.1451 & 5.9981 & 0.3831 & 0.1274 \\
\hline 21 & 7 & 1 & 14.4498 & 5.2617 & 1.7381 & 0.1088 \\
\hline 22 & 3 & 1 & 24.7057 & 4.4770 & 0.0352 & 0.1818 \\
\hline 22 & 4 & 1 & 20.6325 & 5.4021 & 0.1707 & 0.1613 \\
\hline 22 & 5 & 1 & 19.6620 & 4.9759 & 0.3147 & 0.2005 \\
\hline 22 & 7 & 1 & 25.8583 & 7.3746 & 0.7954 & 0.3576 \\
\hline 22 & 15 & 1 & 22.0049 & 9.7484 & 0.8297 & 0.2798 \\
\hline 28 & 3 & 1 & 6.3630 & 1.1301 & 0.0372 & 0.1303 \\
\hline 28 & 4 & 1 & 6.2272 & 1.1718 & 0.1281 & 0.2226 \\
\hline 28 & 15 & 1 & 12.7274 & 7.2239 & 1.1391 & 0.6584 \\
\hline 43 & 3 & 1 & 29.0401 & 4.5319 & 0.0505 & 0.5172 \\
\hline 43 & 4 & 1 & 29.3971 & 9.5485 & 0.6432 & 0.3236 \\
\hline 43 & 5 & 1 & 26.0545 & 6.3729 & 0.5631 & 0.9658 \\
\hline 43 & 7 & 1 & 29.4153 & 15.3730 & 2.4781 & 0.0902 \\
\hline 43 & 15 & 1 & 35.1837 & 15.5455 & 0.9446 & 0.0106 \\
\hline
\end{tabular}


Extended Data Table 4 | Immunoglobulin gene usage of S-binding monoclonal antibodies

\begin{tabular}{|c|c|c|c|c|c|c|c|c|c|}
\hline \multirow[b]{2}{*}{ Name } & \multirow[b]{2}{*}{$\begin{array}{l}\text { Native } \\
\text { isotype }\end{array}$} & \multicolumn{4}{|c|}{ Heavy Chain } & \multicolumn{4}{|c|}{ Light Chain } \\
\hline & & Gene usage & $\begin{array}{l}\text { Nucleotide } \\
\text { mutations }\end{array}$ & $\begin{array}{c}\text { AA } \\
\text { mutations }\end{array}$ & HCDR3 & Gene usage & $\begin{array}{l}\text { Nucleotide } \\
\text { mutations }\end{array}$ & $\begin{array}{c}\text { AA } \\
\text { mutations }\end{array}$ & LCDR3 \\
\hline $07.1 \mathrm{~A} 11$ & $\lg M$ & VH3-15 DH1-7 JH4 & $4 / 283=0.0141$ & 4 & TTGWFTGTYGDYFDY & VK1-33 JK4 & $1 / 267=0.0037$ & 1 & QQYDNLPPT \\
\hline 07.1A12 & IgA1 & VH3-49 D2-15 JH4 & $3 / 281=0.0107$ & 3 & TRVKYCSGGSCYGYHFDH & VK3-15 JK3 & $4 / 262=0 / 0.0153$ & 3 & QQYNNWFT \\
\hline 07.1E10 & $\lg \mathrm{G} 1$ & VH4-34 D6-19 JH6 & $5 / 272=0.0184$ & 5 & ARVVIAVAGTYPIQVYYYYGMDV & VK1-27 JK1 & $2 / 266=0.0075$ & 2 & QKYNSAPRT \\
\hline $07.1 \mathrm{E} 11$ & $\lg A 1$ & VH3-30 D3-22 JH6 & $1 / 278=0.0036$ & 1 & AKEEMIEDWGMDV & VL3-1 JL2 & $2 / 265=0.0075$ & 2 & QAWDRSTVV \\
\hline 07.1H09 & $\lg \mathrm{G} 1$ & VH3-66 DH3-10 JH3 & $3 / 275=0.0109$ & 3 & ARDFREGAFDI & VK1-9 JK4 & $0 / 266=0$ & 0 & QQLNSYPPT \\
\hline $07.2 \mathrm{~A} 07$ & $\lg \mathrm{G} 1$ & VH3-21 DH2-21 JH4 & $6 / 277=0.0217$ & 6 & ARAGFVPKRAYCGGDCWYYFDY & VK3-11 JK4 & $0 / 263=0$ & 0 & QQRSNWLT \\
\hline $07.2 \mathrm{~A} 08$ & $\lg 1$ & VH4-4 DH6-19 JH4 & $2 / 275=0.0073$ & 2 & ATDGGWYTFDH & VL3-1 JL2 & $3 / 265=0.0113$ & 3 & QAWGSSTVV \\
\hline $07.2 \mathrm{~A} 10$ & $\lg 1$ & VH4-31 DH3-16 JH3 & $1 / 278=0.0036$ & 1 & ARYPVWGAFDI & VK1-33 JK3 & $3 / 267=0.0112$ & 3 & QHYDNLPPT \\
\hline $07.2 \mathrm{C} 08$ & $\lg 1$ & VH1-58 DH2-15 JH3 & $2 / 275=0.0073$ & 2 & AAAYCSGGSCSDGEDI & VK3-20 JK1 & $5 / 266=0.0188$ & 5 & QQYGSSPWT \\
\hline $07.2 \mathrm{C} 12$ & $\lg G 1$ & VH3-30-3 DH1-26 JH3 & $3 / 277=0.0108$ & 3 & ARARGGSYSGAFDI & VK3-20 JK2 & $1 / 267=0.0037$ & 1 & QQYGSSPMYT \\
\hline $07.3 \mathrm{D} 07$ & $\operatorname{lgG} 1$ & VH3-30-3 DH5-18 JH4 & $3 / 277=0.0108$ & 3 & ARVLWLRGMFDY & VL6-57 JL3 & $2 / 278=0.0072$ & 1 & QSYDISNHWV \\
\hline $07.4 \mathrm{~A} 07$ & $\operatorname{lgG} 1$ & VH3-30-3 DH3-10 JH4 & $3 / 277=0.0108$ & 2 & ARGDYYGSGSYPGKTFDY & VK1-33 JK4 & $1 / 266=0.0038$ & 1 & QQYDNLPLT \\
\hline $07.4 \mathrm{~B} 05$ & $\lg \mathrm{G} 1$ & VH1-69 DH1-26 JH5 & $1 / 277=0.0036$ & 1 & ARGRLDSYSGSYYSWFDP & VK4-1 JK2 & $2 / 283=0.0071$ & 2 & QQYYSTPYT \\
\hline $07.4 \mathrm{C} 10$ & $\lg G 1$ & VH3-23 DH3-22 JH4 & $1 / 276=0.0036$ & 1 & AKNEMAMIVVVITLFDY & VL1-51 JL3 & $2 / 277=0.0072$ & 2 & GTWDRSLSAWV \\
\hline 07.4D09 & $\lg G 1$ & VH4-4 DH2-15 JH4 & $1 / 274=0.0036$ & 1 & ATKYCSGGSCSYFGY & VL2-23 JL3 & $0 / 277=0$ & 0 & CSYAGSSTWV \\
\hline $20.1 \mathrm{~A} 12$ & $\lg 1$ & VH3-30 DH1-26 JH4 & $2 / 277=0.0072$ & 2 & AKGHSGSYRAPFDY & VK3-20 JK2 & $0 / 263=0$ & 0 & QQYGSSYT \\
\hline $20.2 \mathrm{~A} 03$ & $\lg M$ & VH3-33 DH3-10 JH4 & $1 / 278=0.0036$ & 1 & AREAYFGSGSSPDY & VL3-10 JL2 & $2 / 272=0.0074$ & 2 & YSTDSSDNHRRV \\
\hline $20.3 \mathrm{C} 08$ & $\operatorname{lgG} 1$ & VH3-7 DH3-22 JH4 & $1 / 278=0.0036$ & 1 & AREGTYYYDSSAYYNGGLDY & VL3-10 JL2 & $2 / 268=0.0075$ & 2 & YSTDSGGNPQGV \\
\hline 20.3D12 & $\lg G 1$ & VH3-33 DH1-1 JH4 & $7 / 278=0.0252$ & 7 & ATEPVQLEPEVRLDY & VL3-10 JL1 & $1 / 272=0.0037$ & 1 & YSTDSSGNHRRL \\
\hline $20.3 \mathrm{~F} 12$ & $\operatorname{lgG} 1$ & VH3-7 DH4-11 JH4 & $0 / 278=0$ & 0 & ARDQGVTTGPFDY & VL3-1 JL2 & $1 / 265=0.0038$ & 1 & QAWDSSTVV \\
\hline $22.1 \mathrm{~A} 11$ & $\lg 3$ & VH3-30-3 DH3-16 JH4 & $0 / 278=0$ & 0 & ARDLVVWEELAGGY & VL3-10 JL3 & $2 / 270=0.0074$ & 2 & YSTDSSGNHGV \\
\hline 22.1B04 & $\lg G 1$ & VH3-30 DH2-15 JH4 & $4 / 279=0.0143$ & 3 & AKQGGGTYCGGGSCYRGYFDY & VK1-33 JK4 & $2 / 266=0.0075$ & 2 & QQYDNLPLT \\
\hline $22.1 \mathrm{~B} 08$ & IgA1 & VH1-46 DH4-17 JH3 & $16 / 278=0.0576$ & 15 & ARDPRVPAVTNVNDAFDL & VK3-11 JK2 & $5 / 267=0.0187$ & 5 & QQRSNRPPRWT \\
\hline 22.1B12 & $\operatorname{lgG} 1$ & VH3-53 DH3-10 JH4 & $8 / 273=0.0293$ & 5 & ARSHLEVRGVFDN & VK4-1 JK2 & $1 / 282=0.0035$ & 1 & QQYYSTPCS \\
\hline $22.1 \mathrm{C} 02$ & $\lg 1$ & VH3-20 DH7-27 JH4 & $5 / 275=0.0182$ & 4 & ARGTGAADY & VK3-20 JK2 & $6 / 266=0.0226$ & 6 & QQYGRSPYT \\
\hline 22.1E07 & IgA1 & VH3-33 DH4-17 JH4 & $3 / 278=0.0108$ & 3 & AREGVYGDIGGAGLDY & VL3-10 JL1 & $4 / 272=0.0147$ & 2 & YSTDSSVNGRV \\
\hline 22.1E11 & $\lg 1$ & VH3-30 DH2-15 JH4 & $2 / 274=0.0073$ & 2 & AKMGGVYCSAGNCYSGRLEY & VK1-33 JK3 & $0 / 263=0$ & 0 & QQYDNLLT \\
\hline $22.1 \mathrm{G} 10$ & $\lg 1$ & VH4-59 DH2-21 JH5 & $12 / 275=0.0436$ & 11 & ARETVNNWVDP & VK4-1 JK1 & $10 / 282=0.0355$ & 8 & QQYFTTPWT \\
\hline $22.2 \mathrm{~A} 06$ & $\lg 3$ & VH5-51 DH3-3 JH4 & $1 / 277=0.0036$ & 1 & ARREWGGSLGHIDY & VL6-57 JL2 & $4 / 276=0.0145$ & 4 & QSFDSSNVV \\
\hline $22.2 \mathrm{~A} 08$ & $\lg 1$ & $\mathrm{VH} 4-59 \mathrm{DH} 2-2 \mathrm{JH} 6$ & $4 / 274=0.0146$ & 4 & ARGQGVPAALYGMDV & VL1-40 JL2 & $3 / 281=0.0107$ & 3 & QSYDGSLSGSV \\
\hline $22.2 \mathrm{~B} 06$ & $\lg M$ & VH3-53 DH1-1 JH6 & $2 / 275=0.0073$ & 2 & ARDLQLYGMDV & VL3-21 JL2 & $2 / 268=0.0075$ & 2 & QVWDSSSDPVV \\
\hline $22.2 \mathrm{~F} 03$ & $\lg M$ & VH1-18 DH6-13 JH6 & $1 / 277=0.0036$ & 1 & ARVPGLVGYSSSWYDNEKNYYYYYYGMDV & VL3-25 JL1 & $1 / 270=0.0037$ & 1 & QSADSSGTYV \\
\hline $22.3 \mathrm{~A} 06$ & $\operatorname{lgG} 1$ & VH3-23 DH5-18 JH5 & $2 / 277=0.0072$ & 2 & AKADTAMAWYNWFDP & VK3-11 JK4 & $3 / 264=0.0114$ & 3 & QHRSNWPLT \\
\hline 22.3A11 & $\operatorname{lgG} 1$ & VH4-34 DH7-27 JH2 & $1 / 270=0.0037$ & 1 & ARVWVRWWYFDL & VL3-21 JL1 & $4 / 272=0.0147$ & 4 & QVWDNSSDQPNYV \\
\hline 22.3A12 & $\lg G 1$ & VH1-46 DH2-21 JH4 & $4 / 275=0.0145$ & 4 & ASSLPARGGVPGRLNY & VL1-51 JL2 & $3 / 277=0.0108$ & 3 & GTWDSSLSVVV \\
\hline 22.3D11 & $\lg G 1$ & VH5-51 D4-17 JH5 & $1 / 278=0.0036$ & 1 & ARHHLDYDDYVGHWFDP & VL1-40 JL3 & $0 / 281=0$ & 0 & QSYDSSLSGSGV \\
\hline 22.3F08 & $\lg G 1$ & VH1-18 DH2-2 JH6 & $4 / 275=0.0145$ & 4 & ASCPRRPAAIGDYYGMDV & VL3-21 JL7 & $1 / 271=0.0037$ & 1 & QVWDSSSYHAV \\
\hline
\end{tabular}

*V-region nonsynonymous nucleotide substitutions 


\section{Article}

Extended Data Table 5 | Template switch sequences, constant region primers and isotype-specific internal constant region sequences for bulk BCR sequencing and processing

\begin{tabular}{ll}
\hline Template switch sequences & \\
\hline TS-shift0 & TACGGG \\
TS-shift1 & ATACGGG \\
TS-shift2 & TCTACGGG \\
TS-shift3 & GAATACGGG \\
TS-shift4 & GATCTACGGG \\
& \\
\hline Constant region primers & \\
\hline Human-IGHM & GAATTCTCACAGGAGACGAGG \\
Human-IGHD & TGTCTGCACCCTGATATGATGG \\
Human-IGHA & GGGTGCTGYMGAGGCTCAG \\
Human-IGHE & TTGCAGCAGCGGGTCAAGG \\
Human-IGHG & CCAGGGGGAAGACSGATG \\
Human-IGK & GACAGATGGTGCAGCCACAG \\
Human-IGL & AGGGYGGGAACAGAGTGAC
\end{tabular}

Isotype-specific internal constant region sequences

Human-IGHA-InternalC

Human-IGHD-InternalC

Human-IGHE-InternalC

Human-IGHG-InternalC

Human-IGHM-InternalC

Human-IGKC-IGKJ-InternalC

Human-IGLC-1-InternalC

Human-IGLC-2-InternalC

Human-IGLC-3-InternalC

Human-IGLC-4-InternalC

GGCTGGTCGGGGATGC

GAGCCTTGGTGGGTGC

GGCTCTGTGTGGAGGC

GGCCCTTGGTGGARGC

GGGCGGATGCACTCCC

TTCGTTTRATHTCCAS

TGGGGTTGGCCTTGGG

AGGGGGCAGCCTTGGG

YRGCCTTGGGCTGACC

GCTGCCAAACATGTGC 
Extended Data Table 6 | Processing of bulk sequencing BCR reads

\begin{tabular}{|c|c|c|c|c|c|c|}
\hline \multirow[b]{2}{*}{ Participant } & \multirow[b]{2}{*}{ Sample } & \multirow[b]{2}{*}{$\begin{array}{l}\text { Cell } \\
\text { Count }\end{array}$} & \multicolumn{4}{|c|}{ Sequence Count } \\
\hline & & & Input & Preprocessed & $\begin{array}{l}\text { Post-QC } \\
\text { Productive } \\
\text { Heavy Chains }\end{array}$ & $\begin{array}{l}\text { Unique } \\
\text { Heavy Chain } \\
\text { VDJs }\end{array}$ \\
\hline 07 & d28 blood plasmablast & 8361 & 2307288 & 8294 & 6031 & 3014 \\
\hline 20 & d28 blood plasmablast & 6136 & 2068139 & 2320 & 1453 & 951 \\
\hline 22 & d28 blood plasmablast & 15496 & 1801330 & 16126 & 13733 & 6266 \\
\hline 07 & d28 lymph node germinal centre & 25754 & 1104539 & 2429 & 1700 & 1211 \\
\hline 22 & d28 lymph node germinal centre & 10236 & 2117620 & 552 & 364 & 268 \\
\hline
\end{tabular}




\section{Reporting Summary}

Nature Research wishes to improve the reproducibility of the work that we publish. This form provides structure for consistency and transparency in reporting. For further information on Nature Research policies, see Authors \& Referees and the Editorial Policy Checklist.

\section{Statistics}

For all statistical analyses, confirm that the following items are present in the figure legend, table legend, main text, or Methods section.

n/a Confirmed

$\square \bigotimes$ The exact sample size $(n)$ for each experimental group/condition, given as a discrete number and unit of measurement

$\square$ \A statement on whether measurements were taken from distinct samples or whether the same sample was measured repeatedly

$\square$ The statistical test(s) used AND whether they are one- or two-sided

$\square$ Only common tests should be described solely by name; describe more complex techniques in the Methods section.

$\bigotimes \square$ A description of all covariates tested

$\bigotimes \square$ A description of any assumptions or corrections, such as tests of normality and adjustment for multiple comparisons

$\square$ A full description of the statistical parameters including central tendency (e.g. means) or other basic estimates (e.g. regression coefficient)

$\triangle$ AND variation (e.g. standard deviation) or associated estimates of uncertainty (e.g. confidence intervals)

$\varnothing$ For null hypothesis testing, the test statistic (e.g. $F, t, r$ ) with confidence intervals, effect sizes, degrees of freedom and $P$ value noted

Give P values as exact values whenever suitable.

Х $\square$ For Bayesian analysis, information on the choice of priors and Markov chain Monte Carlo settings

Х $\square$ For hierarchical and complex designs, identification of the appropriate level for tests and full reporting of outcomes

Х Estimates of effect sizes (e.g. Cohen's $d$, Pearson's $r$ ), indicating how they were calculated

our web collection on statistics for biologists contains articles on many of the points above.

\section{Software and code}

Policy information about availability of computer code

Data collection Flow cytometry data were acquired using SpectroFlo software v2.2.

Data analysis Flow cytometry data were analyzed using FlowJo v10 and Prism v8. ELISA, ELISpot, and neutralization data were analyzed using Prism v8. Sequence data were analyzed using blastn v2.11.0, pRESTO v0.6.2, cd-hit-est v4.8.1, IgBLAST v1.17.1, IMGT/GENE-DB release 202113-2, Change-O v1.0.2, TIgGER v1.0.0, igraph v1.2.5, and SHazaM v1.0.2.

For manuscripts utilizing custom algorithms or software that are central to the research but not yet described in published literature, software must be made available to editors/reviewers. We strongly encourage code deposition in a community repository (e.g. GitHub). See the Nature Research guidelines for submitting code \& software for further information.

\section{Data}

Policy information about availability of data

All manuscripts must include a data availability statement. This statement should provide the following information, where applicable:

- Accession codes, unique identifiers, or web links for publicly available datasets

- A list of figures that have associated raw data

- A description of any restrictions on data availability

Antibody sequences are deposited on GenBank under the following accession numbers: MW926396-MW926407, MW926409-MW926430, MW926432MW926441, MZ292481-MZ292510, available from GenBank/EMBL/DDBJ. Bulk sequencing reads are deposited on Sequence Read Archive under BioProject PRJNA731610. The IMGT/V-QUEST database is accessible at http://www.imgt.org/IMGT_vquest/. Other relevant data are available from the corresponding author upon request. 


\section{Field-specific reporting}

Please select the one below that is the best fit for your research. If you are not sure, read the appropriate sections before making your selection. $\bigotimes$ Life sciences $\quad \square$ Behavioural \& social sciences $\quad \square$ Ecological, evolutionary \& environmental sciences

For a reference copy of the document with all sections, see nature.com/documents/nr-reporting-summary-flat.pdf

\section{Life sciences study design}

All studies must disclose on these points even when the disclosure is negative. $\begin{array}{ll}\text { Sample size } & \text { No statistical methods were used to determine sample size. } 41 \text { total participants were enrolled based on recruitment, of whom } 14 \text { provided } \\ \text { axillary LN samples }\end{array}$

Data exclusions No data were excluded

Replication Human samples were collected from 41 participants. For ELISA, ELISpot, and neutralization assays, results were from one experiment performed in duplicate. Flow cytometry experiments were performed once

Randomization Different experimental groups were not used.

Blinding No blinding was done for convenience; subjective measurements were not made.

\section{Reporting for specific materials, systems and methods}

We require information from authors about some types of materials, experimental systems and methods used in many studies. Here, indicate whether each material, system or method listed is relevant to your study. If you are not sure if a list item applies to your research, read the appropriate section before selecting a response.

\begin{tabular}{l|l} 
Materials \& experimental sys \\
\hline $\mathrm{n} / \mathrm{a}$ & Involved in the study \\
\hline & $\bigotimes$ Antibodies \\
$\square$ & $\square$ Eukaryotic cell lines \\
$\square$ & $\square$ Animals and other organisms \\
$\square$ Clinical data
\end{tabular}

\begin{tabular}{l|l}
\multicolumn{2}{l}{ Methods } \\
\hline n/a & Involved in the study \\
\hline & $\square$ ChIP-seq \\
$\square$ & $\square$ Flow cytometry \\
$\square$ MRI-based neuroimaging
\end{tabular}

\section{Antibodies}

Antibodies used

total Ig (goat polyclonal, Jackson ImmunoResearch 109-005-064), IgG-HRP (goat polyclonal, Jackson ImmuoResearch 109-035-088), IgA-HRP (goat polyclonal, Jackson ImmuoResearch 109-035-011), IgM-HRP (goat polyclonal, Caltag H15007), mouse IgG-HRP (goat polyclonal, Sigma 12-349), murine anti-S mAbs: SARS2-2, SARS2-11, SARS2-16, SARS2-31, SARS2-38, SARS2-57, and SARS2-71 (Diamond laboratory, Washington University School of Medicine), IgG-BV480 (goat polyclonal, Jackson Immunoresearch 109-685-098), PD-1-BB515 (EH12.1, BD Horizon 564494), IgA-FITC (M24A, Millipore CBL114F), CD45-A532 (HI30, Thermo 58-0459-42), Bcl6-PE (K112-91, 561522, BD Pharmingen), CD38-BB700 (HIT2, 566445, BD Horizon), Blimp1-A700 (646702, IC36081N, R\&D), CD19-BV421 (HIB19, 302234), FoxP3-BV421 (206D, 320124), CD20-Pacific Blue (2H7, 302320), CD27BV510 (O323, 302836), CD8-BV570 (RPA-T8, 301038), IgM-BV-605 (MHM-88, 314524), HLA-DR-BV650 (L243, 307650), Ki-67BV711 (Ki-67, 350516), CD19-BV750 (HIB19, 302262), Tbet-BV785 (4B10, 644835), CD3-FITC (HIT3a, 300306), CD71-FITC (CY1G4, 334104), IgD-PE (IA6-2, 348204), CD71-PE (CY1G4, 334106), CXCR5-PE-Dazzle 594 (J252D4, 356928), IgD-PE-Cy5 (IA6-2, 348250), CD4-PerCP (OKT4, 317432), CD14-PerCP (HCD14, 325632), IgD-PerCP-Cy5.5 (IA6-2, 348208), CD38-PE-Cy7 (HIT2, 303516), CD71PE-Cy7 (CY1G4, 334112), CD19-APC (HIB19, 302212), CD4-Spark 685 (SK3, 344658), CD20-APC-Fire750 (2H7, 302358), CD3-APCFire810 (SK7, 344858); all Biolegend.

Validation

All commercial antibodies were validated by their manufacturers as detailed in their product information and titrated in the lab for the indicated assay by serial dilution. We validated mAbs generated in our lab in preliminary ELISAs to SARS-CoV-2 spike, bovine serum albumin, and anti-lg. 


\section{Eukaryotic cell lines}

Policy information about cell lines

Cell line source(s)

Expi293F (Thermo), Vero E6 (CRL-1586, American Type Culture Collection), Vero-TMPRSS2 (Ding laboratory, Washington University School of Medicine), Vero-hACE2-TMPRSS2 (Graham laboratory, VRC/NIH)

Authentication

All cell lines grew and performed as expected. Expression of TMPRSS2 on Vero-TMPRSS2 was validated by flow cytometry [ref. 17]. No additional specific authentication was performed.

Mycoplasma contamination

Vero lines were tested monthly for mycoplasma and were negative. Expi293F lines were not tested.

Commonly misidentified lines (See ICLAC register)

No commonly misidentified cell lines were used

\section{Human research participants}

Policy information about studies involving human research participants

Population characteristics

Recruitment

Ethics oversight
Study participants demographics are detailed in Extended Data Table 1

Study participants were recruited from the St. Louis metropolitan area by the Washington University Clinical Trials Unit. Potential self-selection and recruiting biases are unlikely to affect the parameters we measured.

The study was approved by the Washington University IRB

Note that full information on the approval of the study protocol must also be provided in the manuscript.

\section{Flow Cytometry}

Plots

Confirm that:

ХThe axis labels state the marker and fluorochrome used (e.g. CD4-FITC).

\The axis scales are clearly visible. Include numbers along axes only for bottom left plot of group (a 'group' is an analysis of identical markers).

\All plots are contour plots with outliers or pseudocolor plots.

$\bigotimes$ A numerical value for number of cells or percentage (with statistics) is provided.

\section{Methodology}

Sample preparation

Fine needle aspirates of axillary LNs were flushed from needles with $3 \mathrm{~mL}$ of RPMI supplemented with $10 \% \mathrm{FBS}$ and $100 \mathrm{U} / \mathrm{mL}$ penicillin/streptomycin, followed by three $1 \mathrm{~mL}$ rinses. Red blood cells were lysed with ammonium chloride buffer, washed twice with PBS supplemented with 2\% FBS, 2mM EDTA and immediately used or cryopreserved in 10\% DMSO in FBS.

Blood samples were collected in EDTA tubes, and peripheral blood mononuclear cells (PBMCs) were enriched by density gradient centrifugation over Ficoll 1077 (GE) or Lymphopure (BioLegend). The residual red blood cells were lysed with ammonium chloride lysis buffer, and cells were immediately used or cryopreserved in $10 \%$ dimethylsulfoxide in FBS.

Instrument Cytek Aurora

Software

Flow cytometry data was acquired using Cytek SpectroFlo and analyzed using FlowJo (Treestar) v10.

Cell population abundance

Single cell sorts and bulk sorts directly into lysis buffer were not amenable to post-sort purity analysis.

Gating strategy

Gating strategies are shown in Extended Data Figure 2

$\bigotimes$ Tick this box to confirm that a figure exemplifying the gating strategy is provided in the Supplementary Information. 